$[r \cdot \xi-r \Lambda 1] ص[n$

الأسواق المالية والنمو الاقتصادي في الدول النامية(*)

$$
\begin{aligned}
& \text { مثنى عبد الرذاق الدباغ }
\end{aligned}
$$

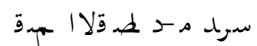

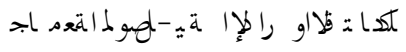

Muthana aldabgh@vahoo.com

$$
\begin{aligned}
& \text { الدكتور مفيد ذنون يونس }
\end{aligned}
$$

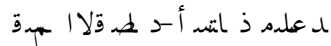

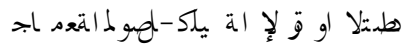

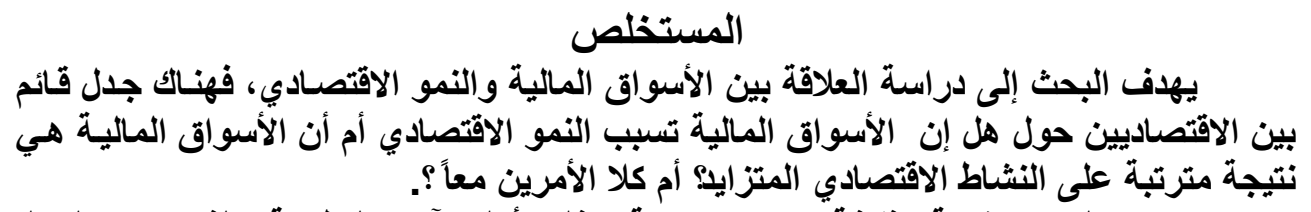

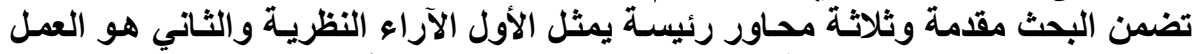

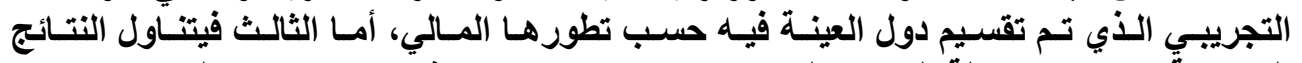

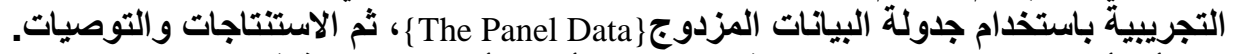

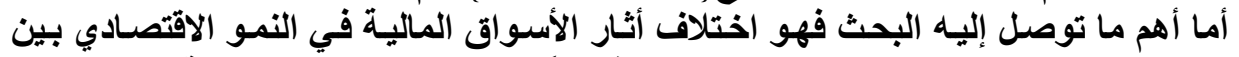

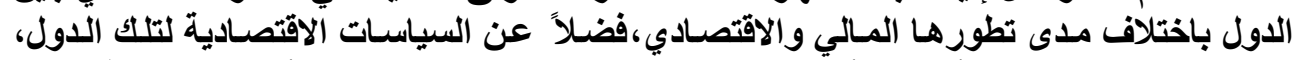

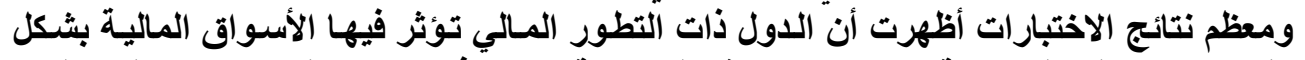

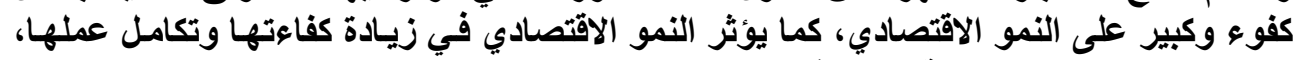

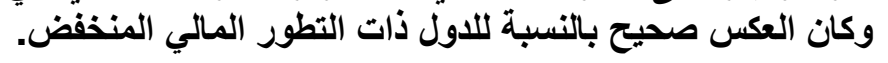

(") البحث مستل من اطروحـة الدكتور اه الموسومة "تأثثير الأسواق المالية في النمو الاقتصـادي لبلدان نامية مختارة"، كلية الادارة والاقتصاد -جامعة الموصل.

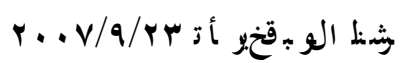

شحبل املاتسلخه أ ز 


\title{
The Financial Markets And Economic Growth In Developing Country
}

\author{
Dr. Mufeed T. Younis \\ Assistant Professor- Dept. of Economics \\ University of Mosul
}

\author{
Dr. Muthana A. Al-Dabbagh \\ Lecturer- Dept. of Economics \\ University of Mosul
}

\begin{abstract}
The current paper aims at studying the relationship between financial markets and economic growth. There is a gradual controversial among economists about a question that say if financial markets may cause economic growth or if financial markets is a real result for the increased economic activity ? or both states.

The paper included an introduction and three basic themes.The first represents theoretical concepts the second is the empirical aspect in which sample states are divided according to their financial development. The third deals with the empirical results by using" The Panel Date" and finally conclusions and recommendations.

The most important results of this paper is the difference of financial markets in economic growth among countries by the difference in its financial and economic development. Also, economic policies for these countries and most results for the tests showed that countries of financial development are being affected by financial markets largely and efficiently on economic growth. Economic growth may affect in increasing the efficiency and the completion of its duty for the financial markets. The opposite is the right for states of reduced financial development.
\end{abstract}

ا بـ أمقدمة

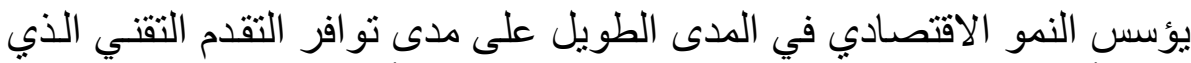

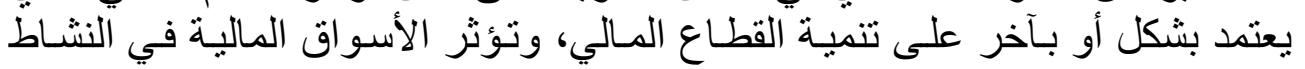

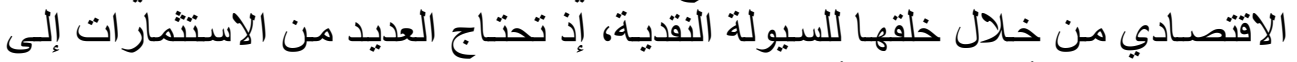

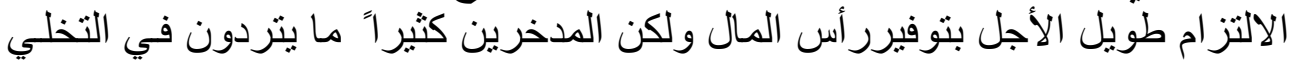

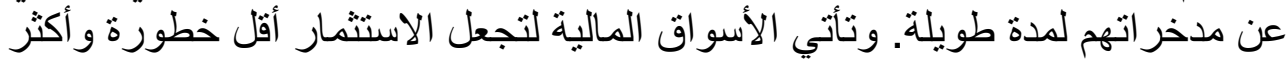

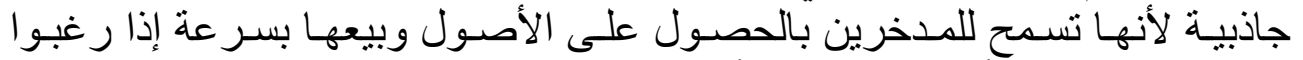

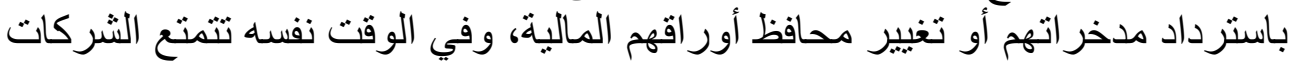

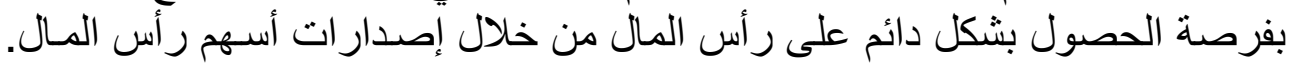

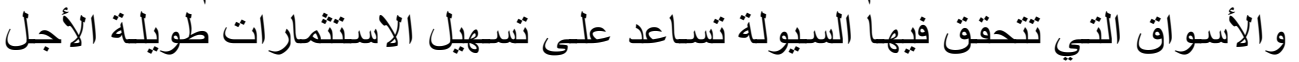
و الأكثر ربحية وأن تحسين تخصيص رأس المال يعزز إقامة النمو الاقتصادي طويل

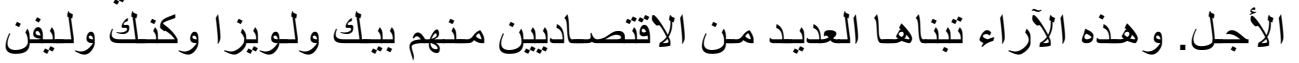

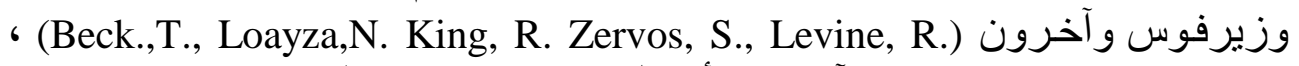

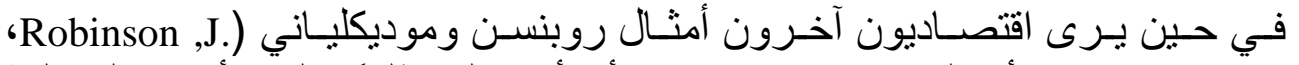

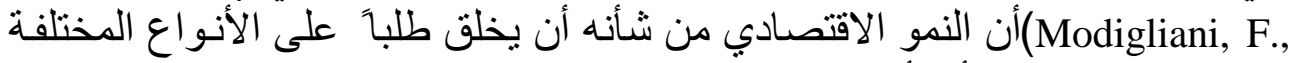

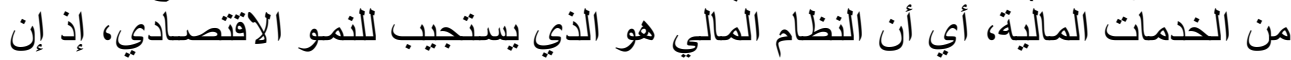

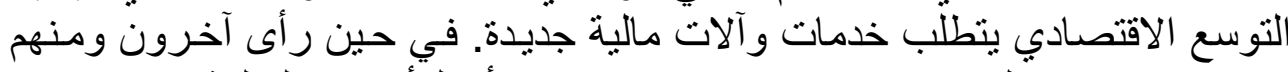
سمث وروبرت ولوكاس (Smith, Robert, Lucas) أن للأسـواق المالية دور محدود 


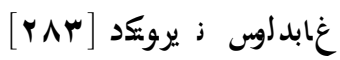

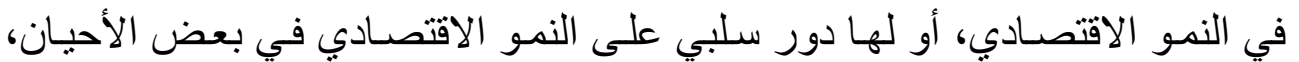

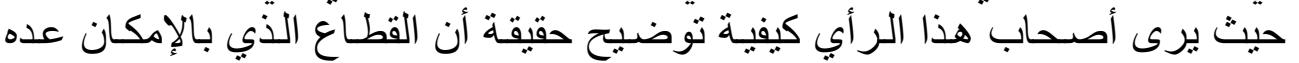

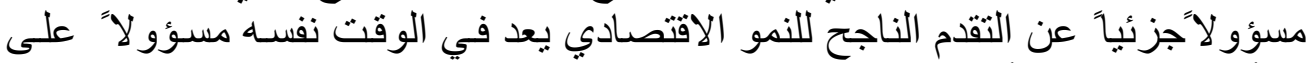
الأقل في تحفيز الأزمات التي تخفض النف النئ النمو الاقتصادي.

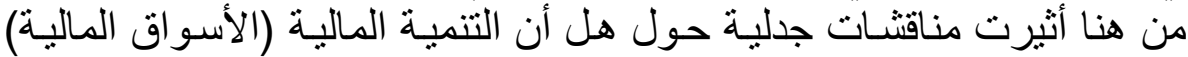

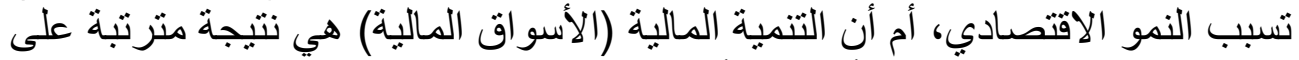

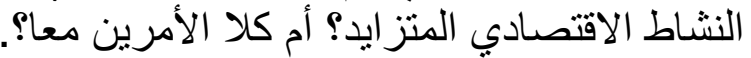
وتنطلق فرضية البحث من المشكلة السابقة وتنقسم إلى الإن:

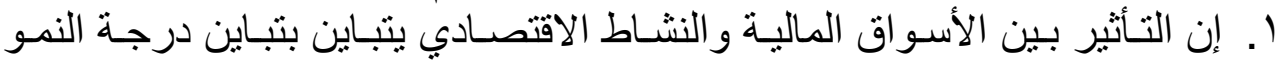

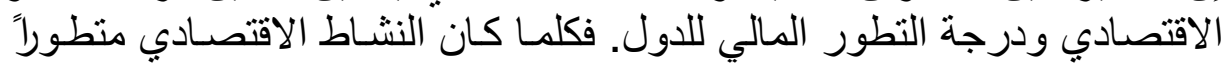

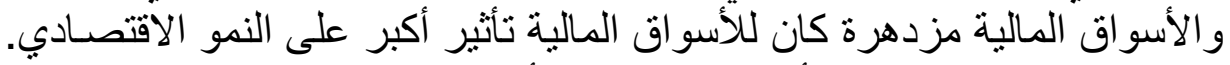

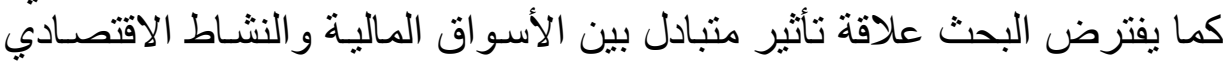

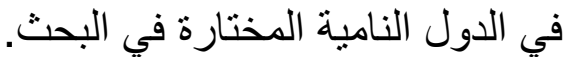

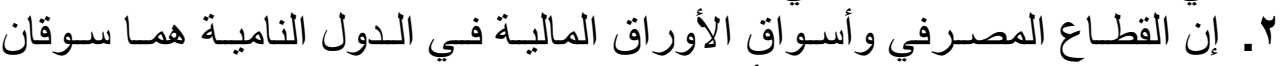
متكاملان وليسا هُتنافسين في تأثير هما على الألى النمو الاقتصادي.

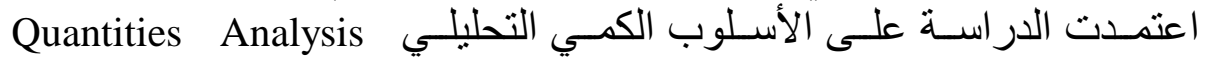
Approach

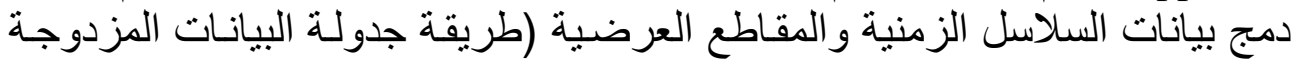
(The Panel Data\}

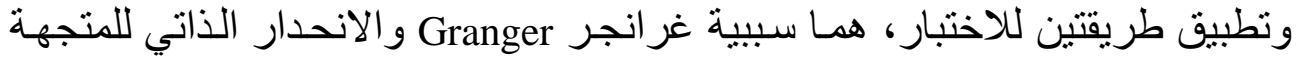

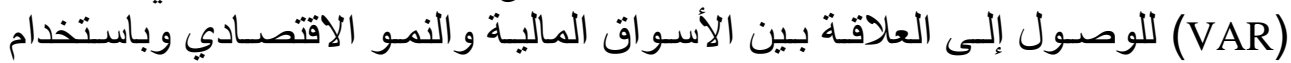

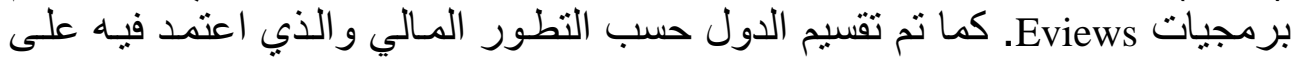

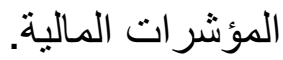
وتم الاعتماد على العديد من المصادر العلمية من البحوث و الكتب في الجانبيين

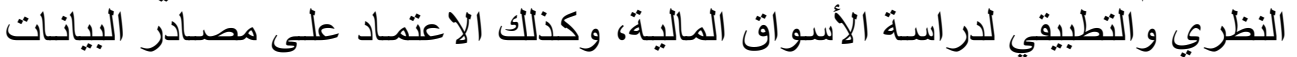

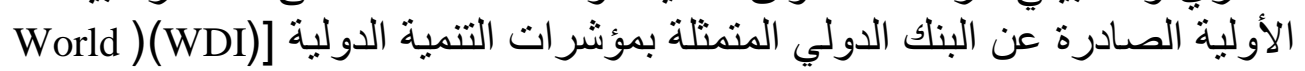
.](Development Indicator

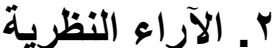

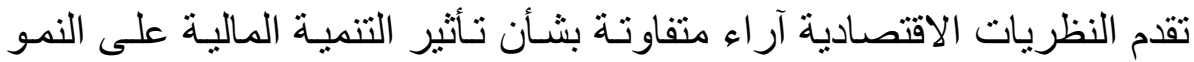

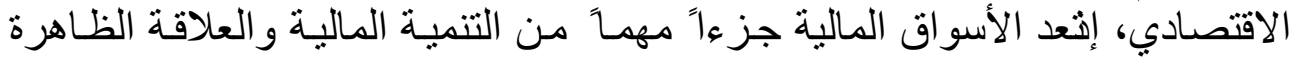

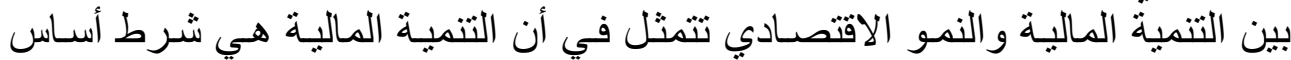

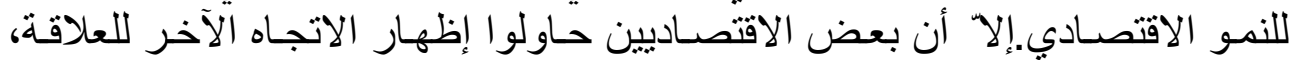

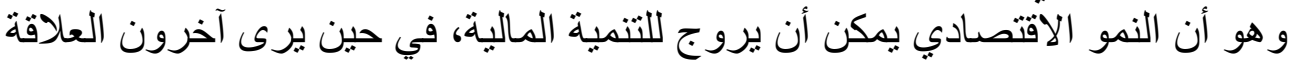

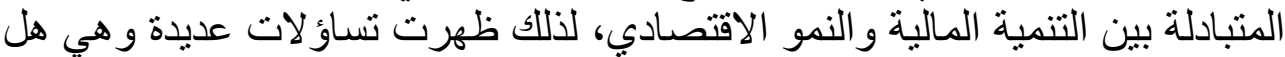

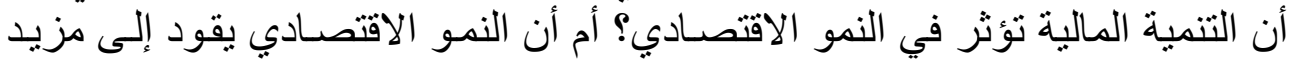


من التنمية المالية؟ أم أن العلاقة السببية تسير في كلا الاتجـاهين لذللك سيتم مناقثـة

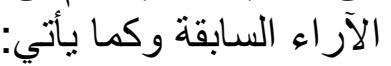
r-1 التنمية المالية تؤدي إلى النى النمو الاقتصادي

Financial Development Causes Economic Growth

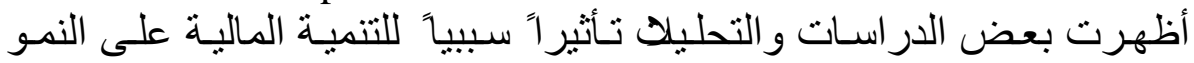

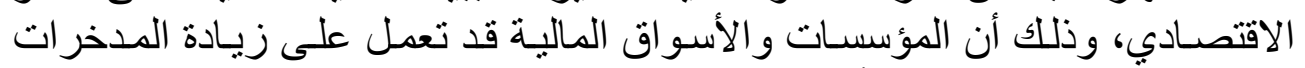

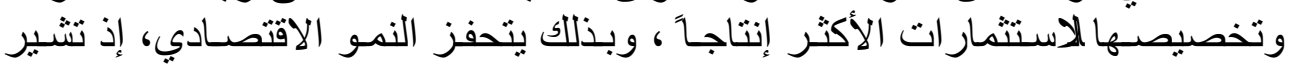

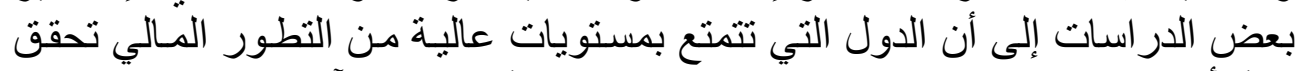

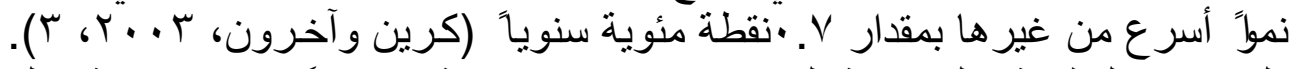

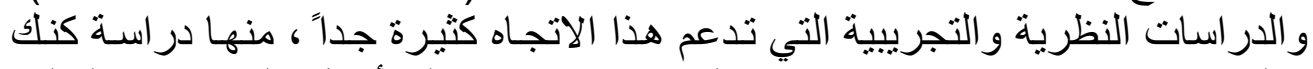

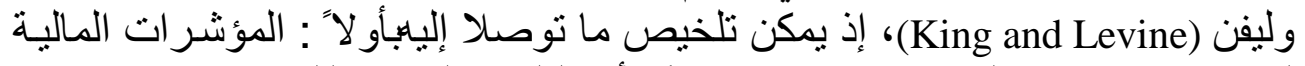

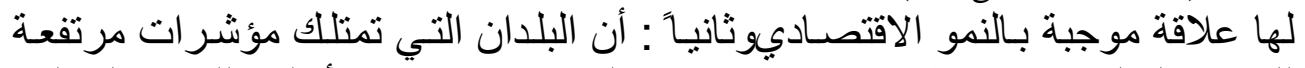

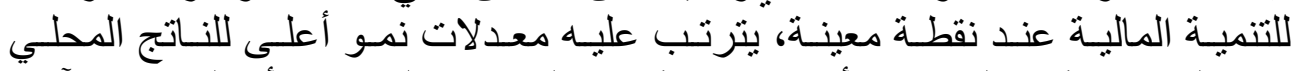

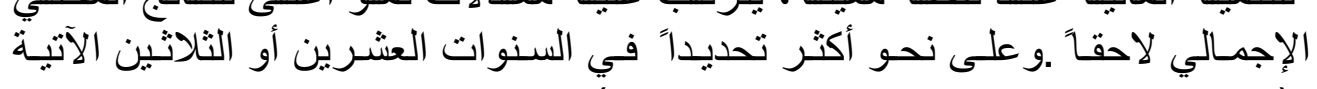
.(King, R., and Levine, R., 1993, 734-735) وأظهر (Levine, R., and Zervos, S., A, 1996, 29) بـأن الأسـواق

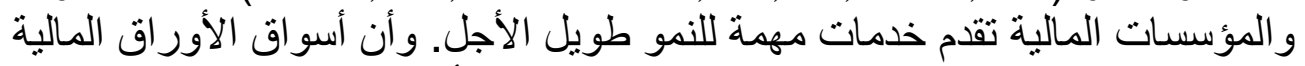

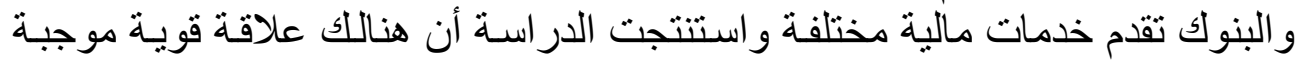

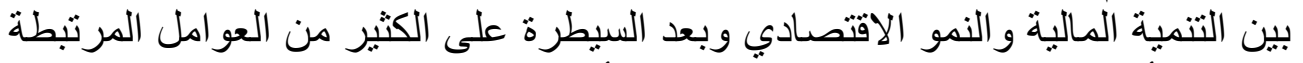

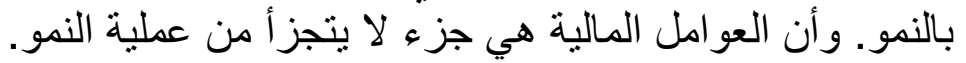

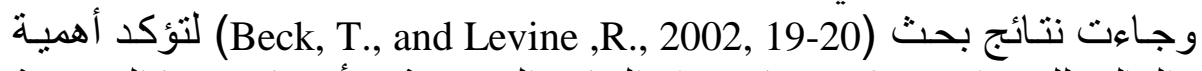

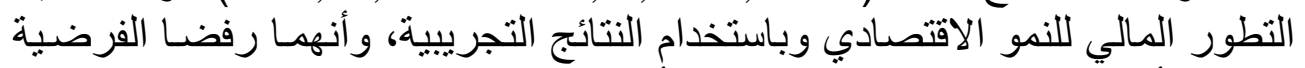

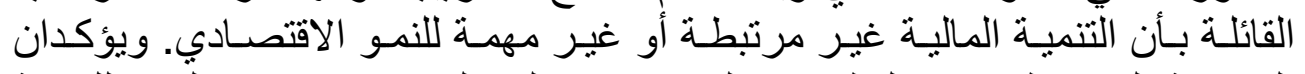

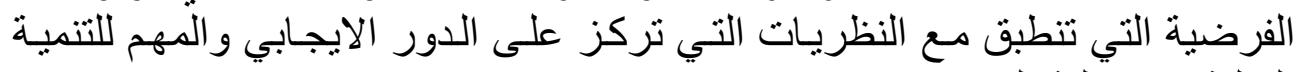

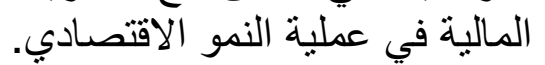

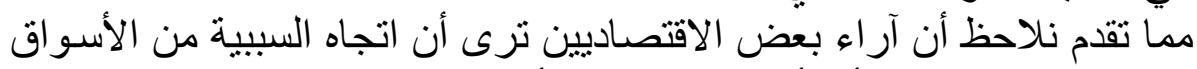

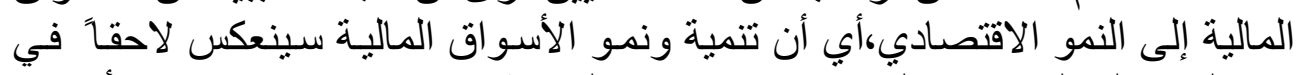

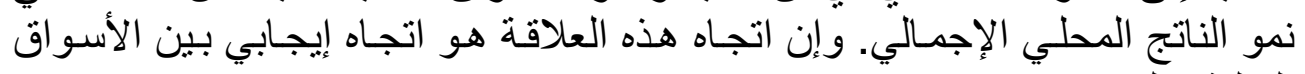
المالية والنمو الاقتئصادي.

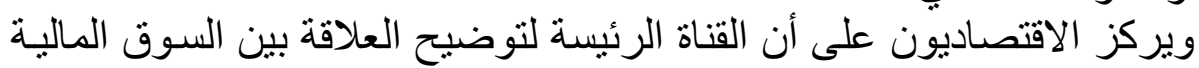

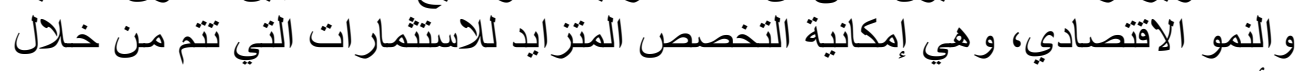

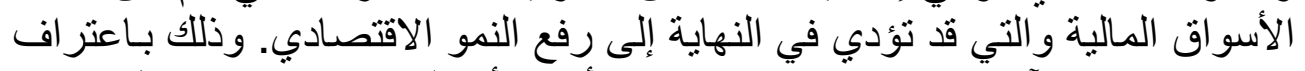

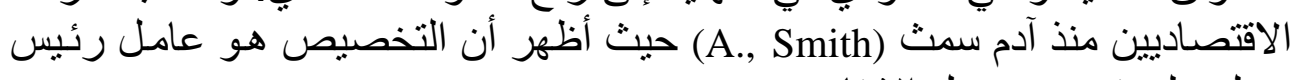
يعمل على تحسين معدل الإنتاج.

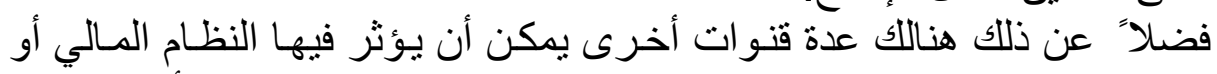

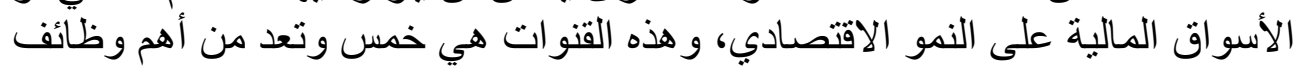




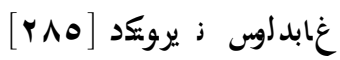

النظام المالي التي تؤثر في النمو الاقتصـادي وتؤدي إلى رفع كفاءة توزيع الموارد

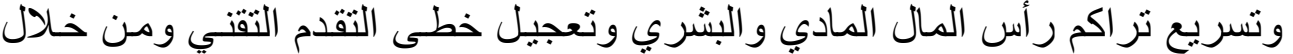
الثكل إيكن توضيح هذه الصورة.

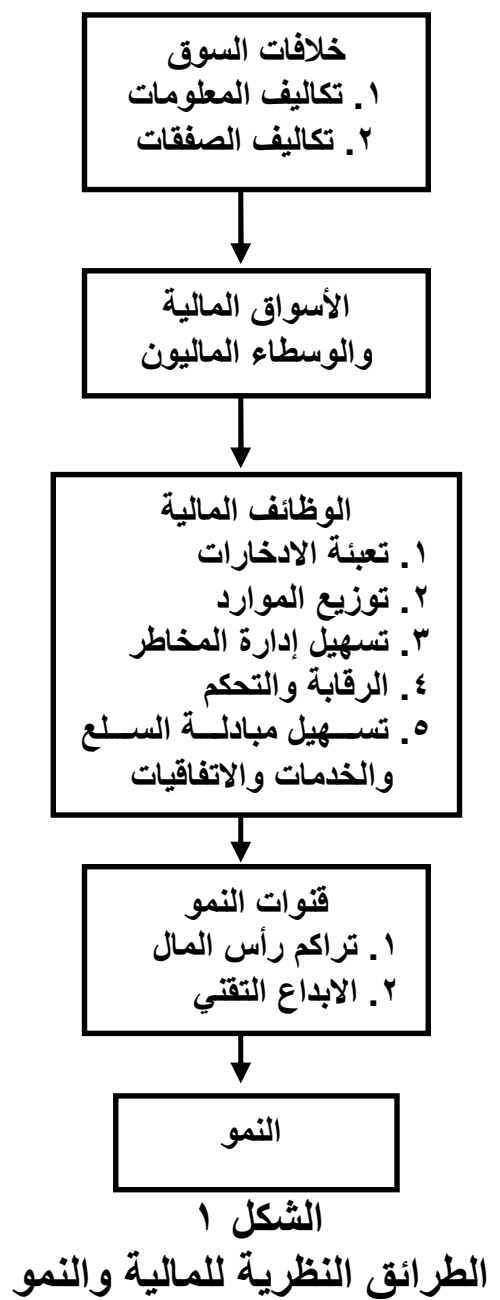

Source: Levine, 1996, Financial Development and Economic Growth, View and Agenda, working paper, 1678, World Bank, 81.

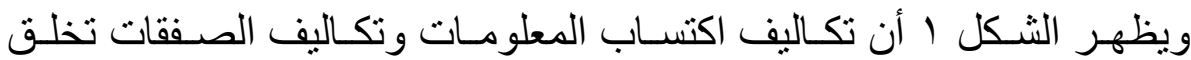

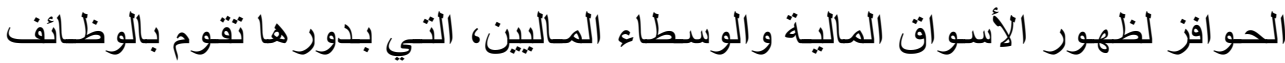

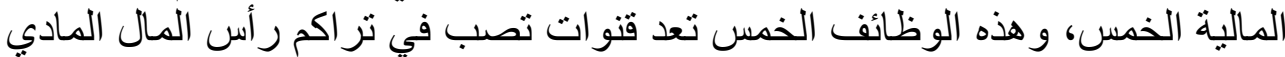

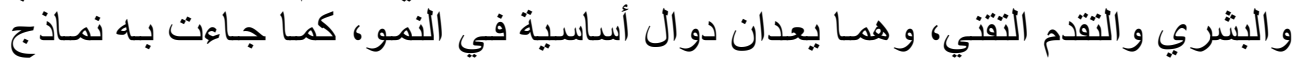
النمو.

و وأظهرت آراء أخرى لاقتصـاديين أن للتتميـة المالية أو الأسواق المالية دوراً

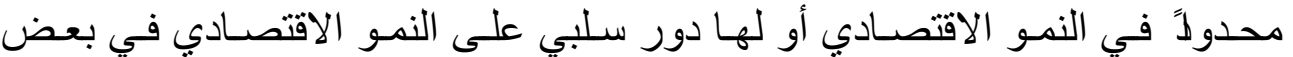




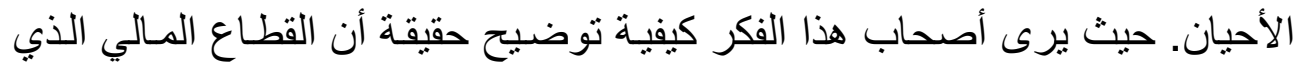

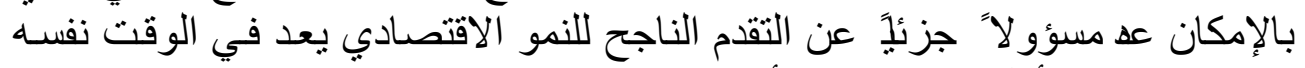

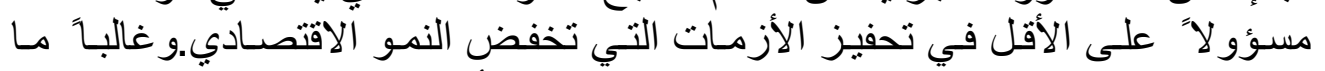

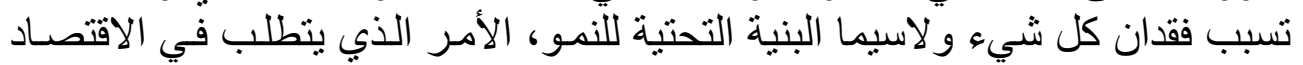

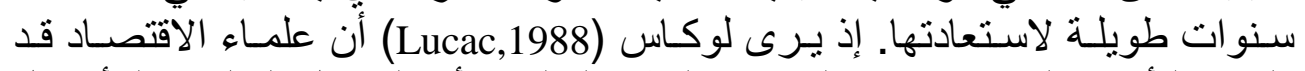

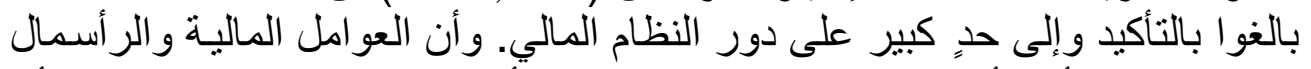

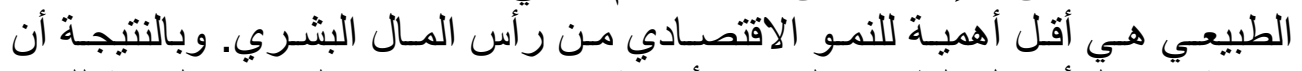

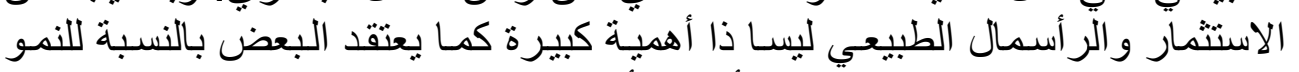

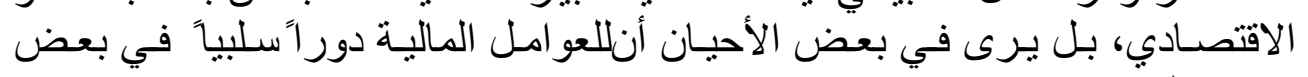

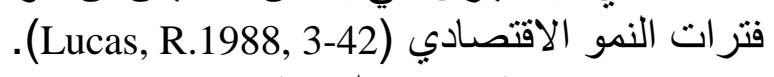

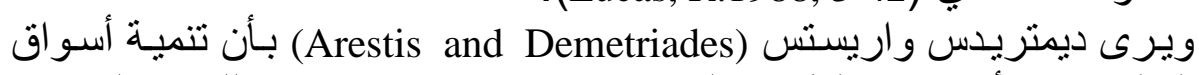

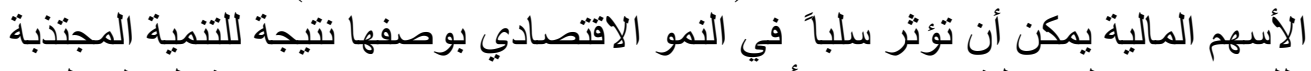

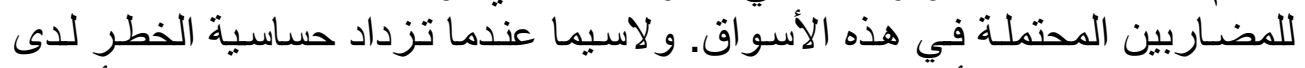

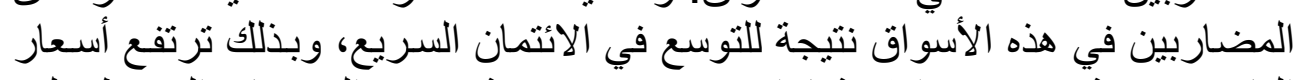

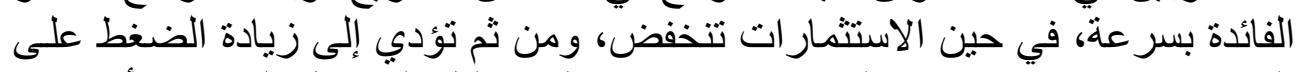

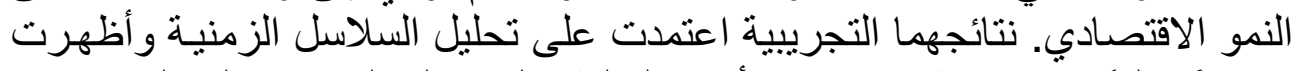

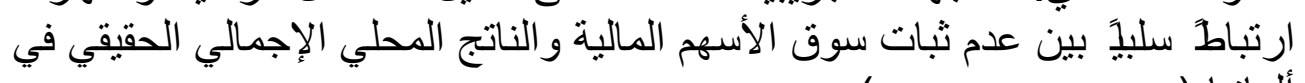
ألمانيا (Haas, 2001, 43-44).

\section{r r r النمو الاقتصادي يسبب التتمية المالية}

\section{Economic Growth Causes Financial Development}

ترى نظريات وبحوث أخرى ذات وجهات نظر مختلفة بـأن النمو الاقتصـادي

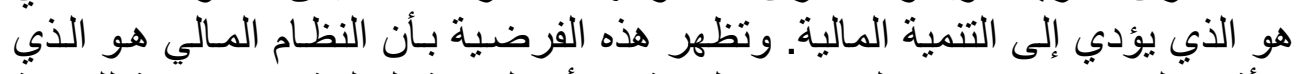

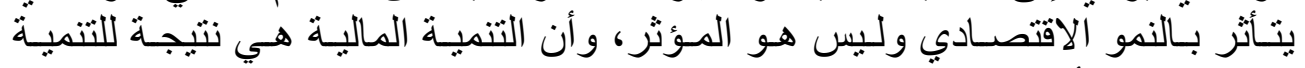

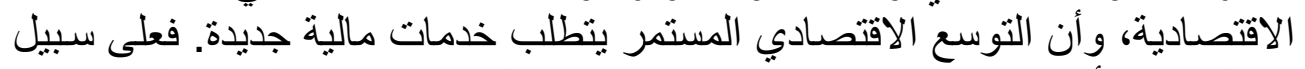

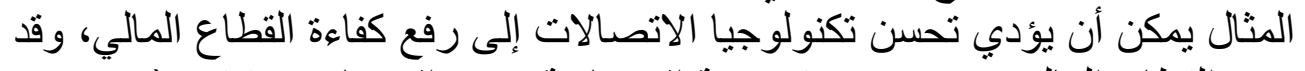

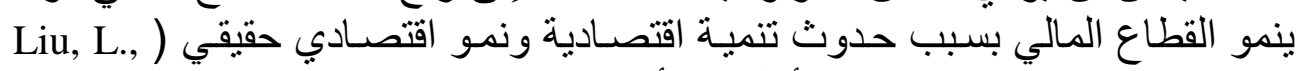
(J. دون (and Garcia ,V., 1999, 35 Robinson,1952)

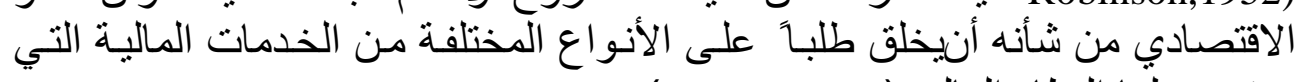
يستجيب لُها النظام المالي (Brandl, M., 2).

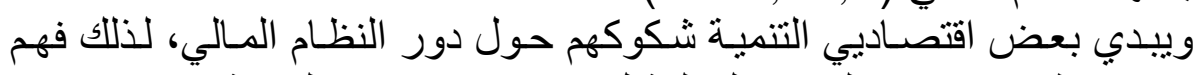

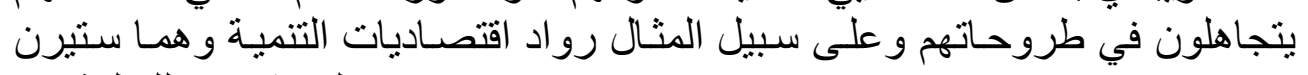

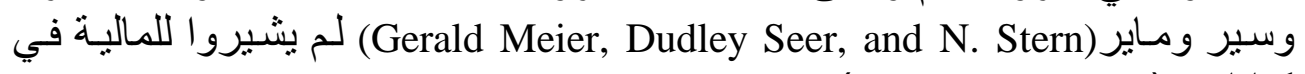
كتاباتهم (Levine, R.,1996,1). 


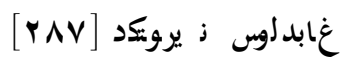

r r r العلاقة المتبادلة بين التنمية المالية والنمو الاقتصادي The Mutual Relationships between Financial Development and Economic Growth.

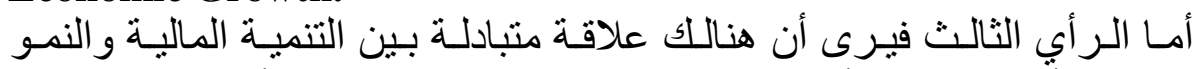

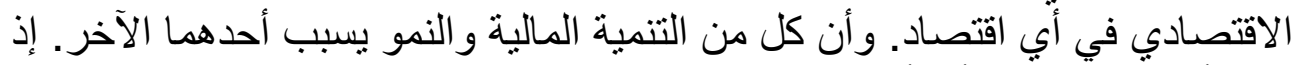

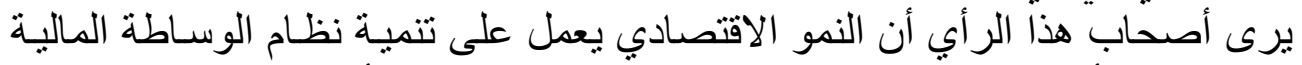

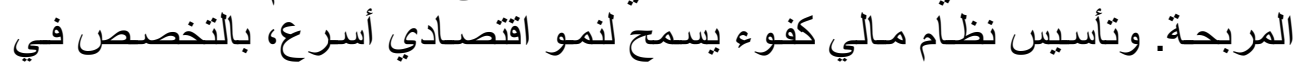

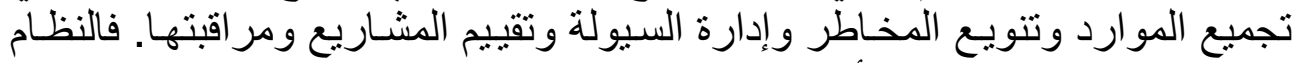

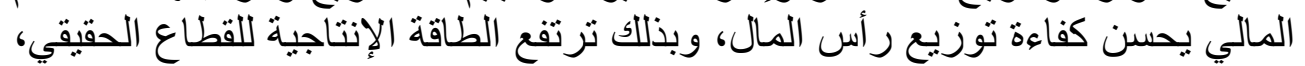

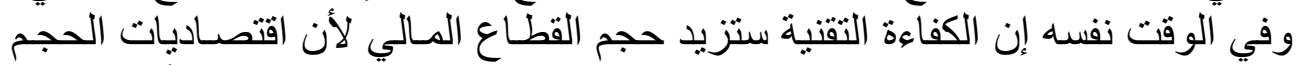

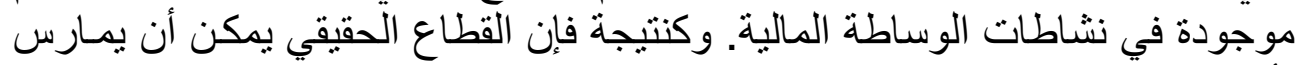

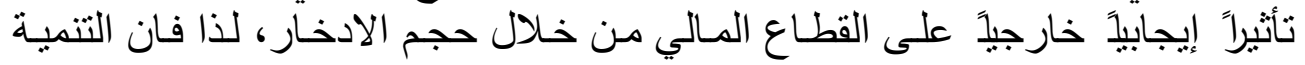

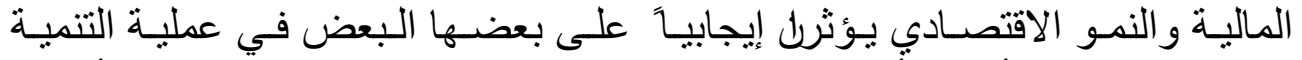

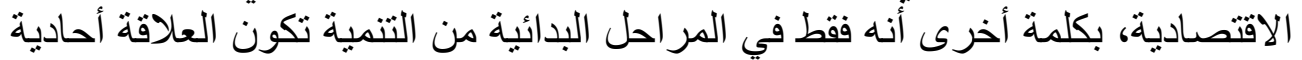

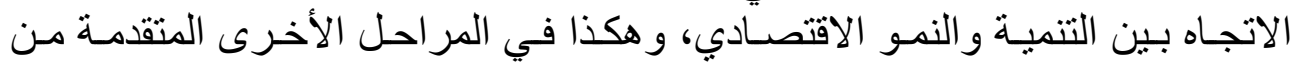

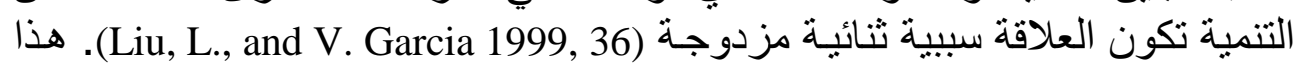

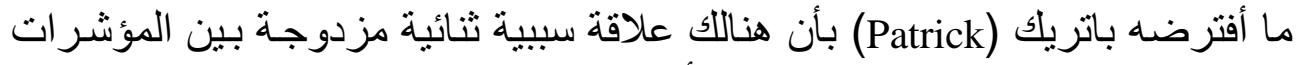

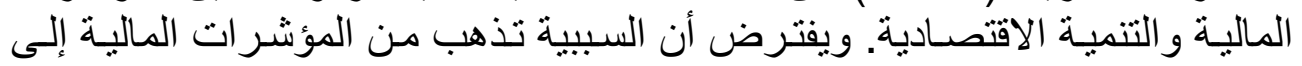

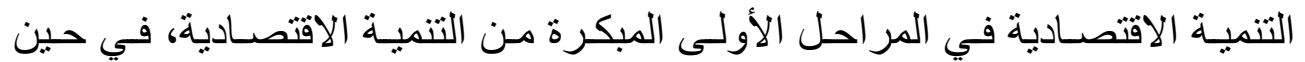

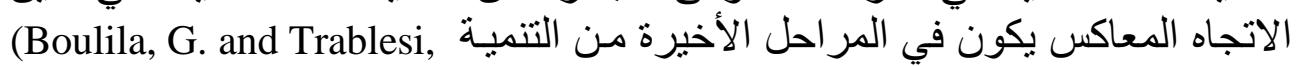
.M. 2002, 4)

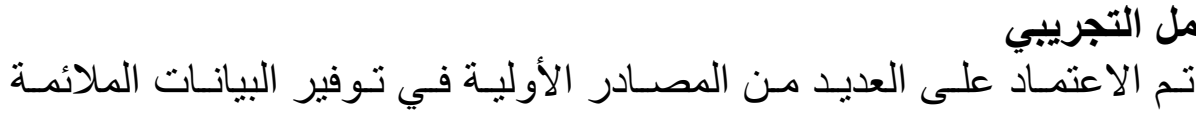

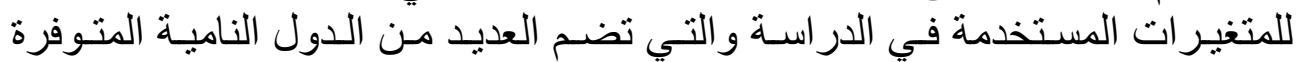

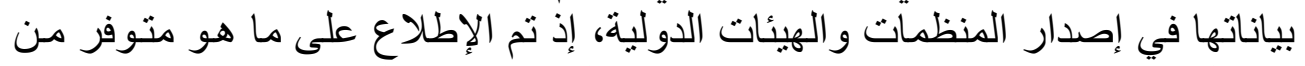

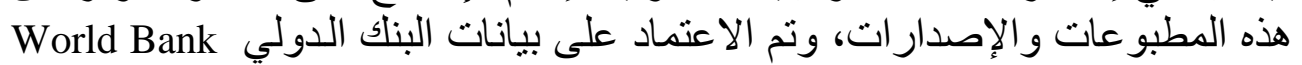
Indicator (WDI)

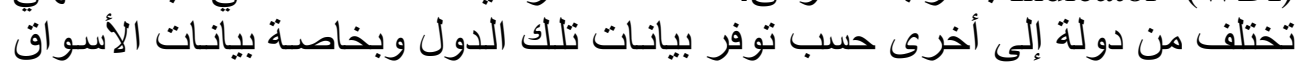

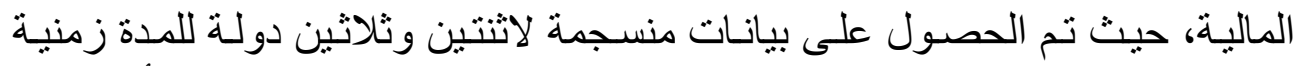

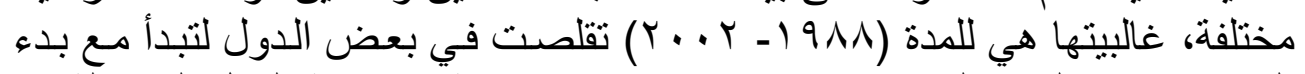

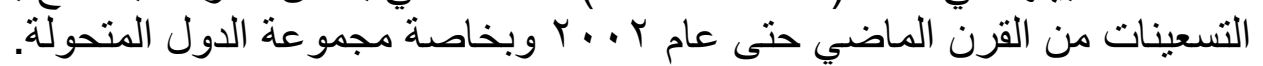

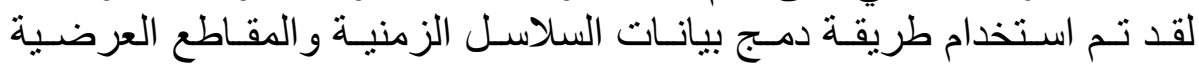
(Pooling Cross-Section and Time Series Data Models)

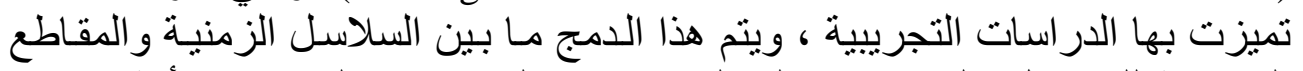

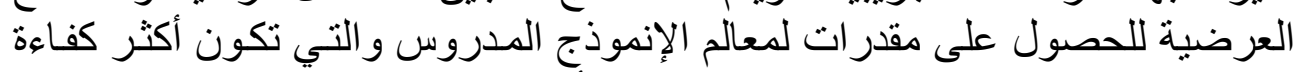

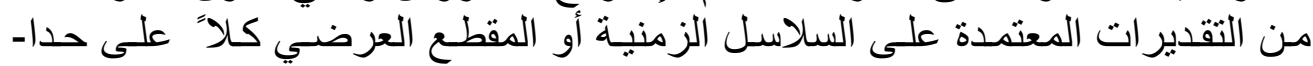


و تتضمن الأساليب المستخدمة لدمج البيانات على مستوى المعادلة المنفردة استخدام

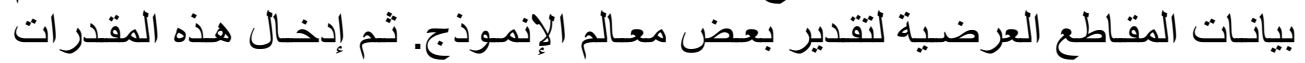

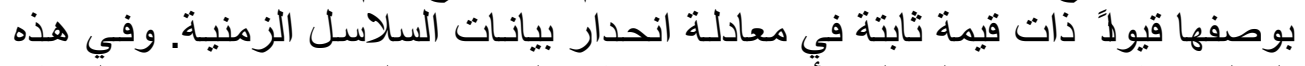

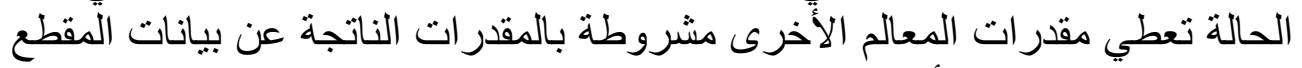

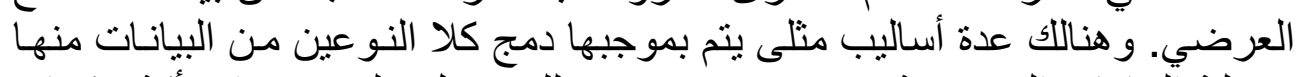

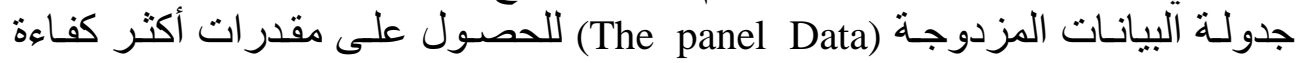

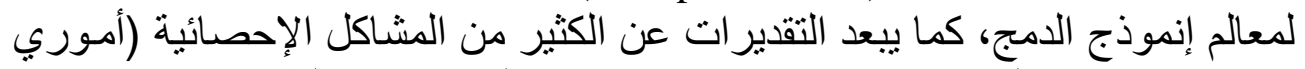

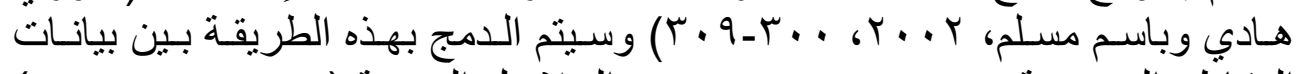

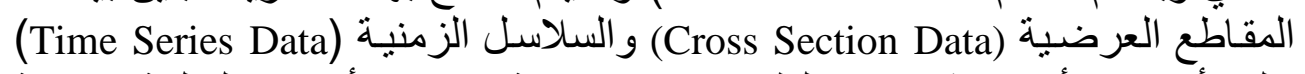

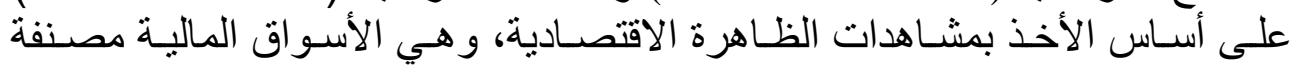

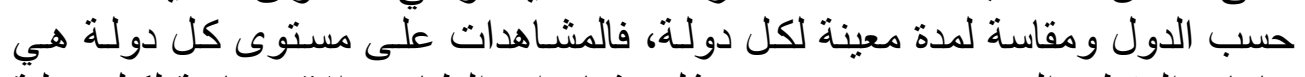

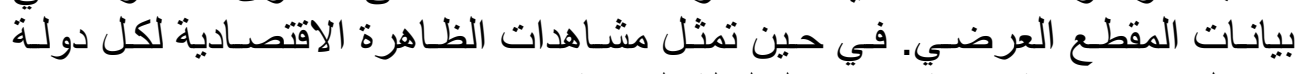

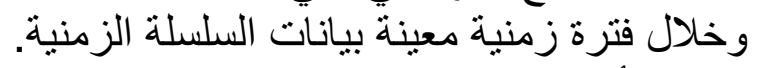

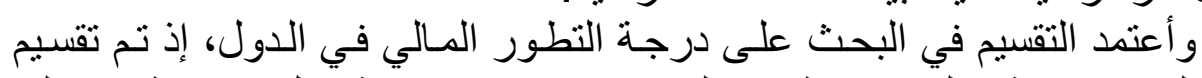

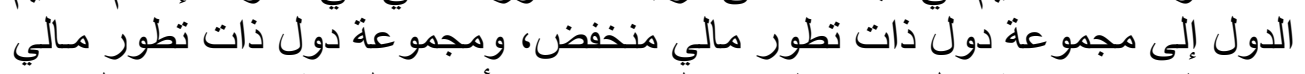

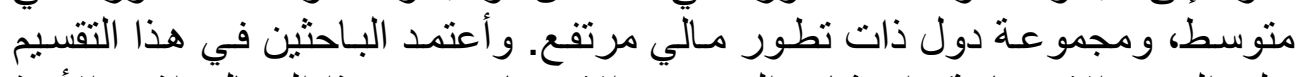

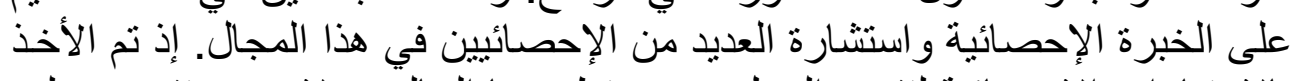

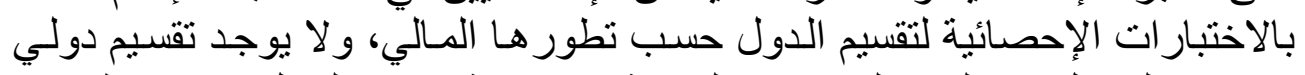

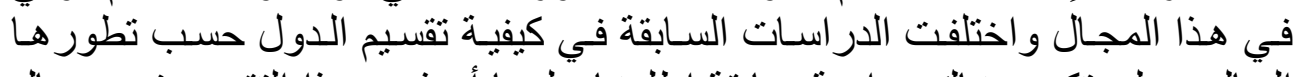

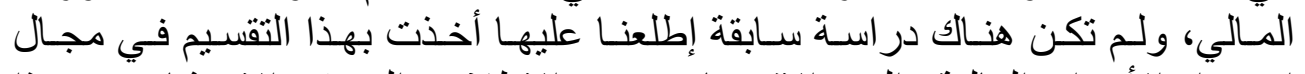

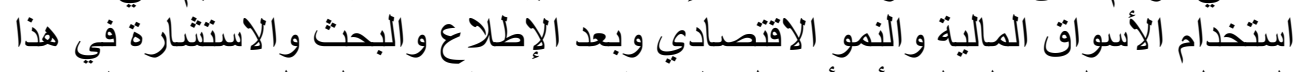

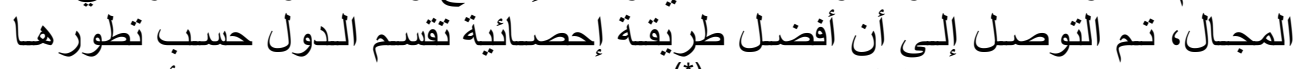

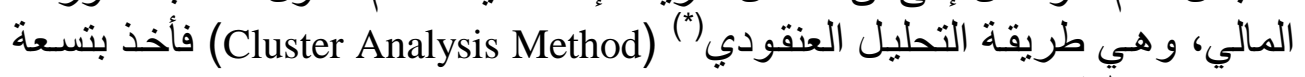

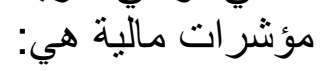

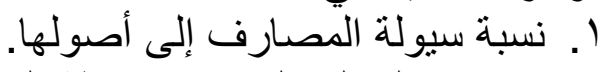
r. الائتمان المحلي المجهز بواسطة المصارف كنسبة من الناتج المحلي الإجمالي.

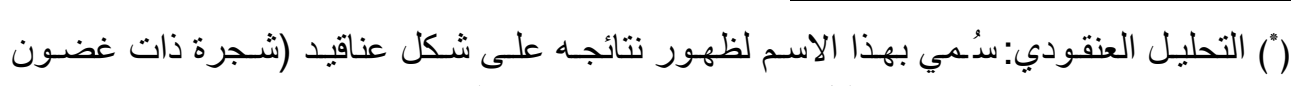

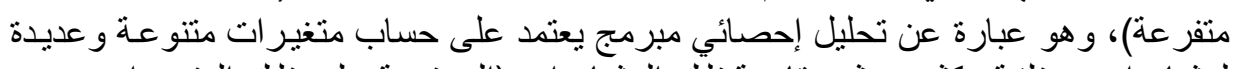

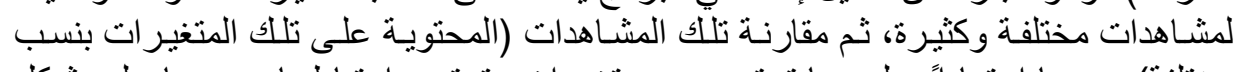

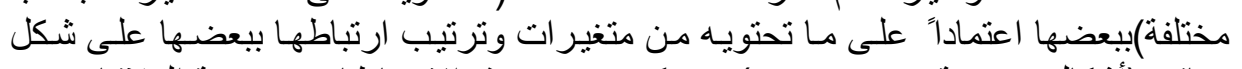

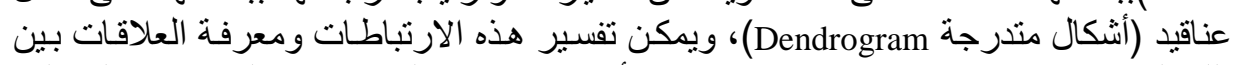

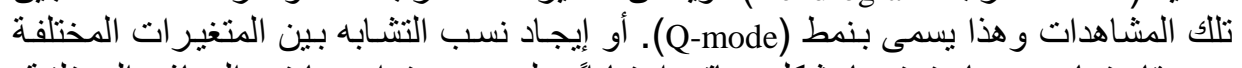

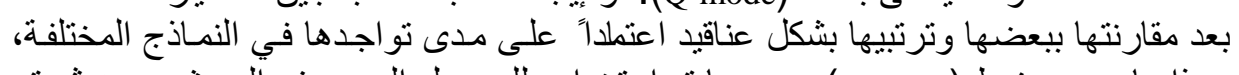

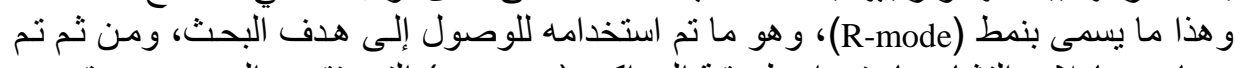

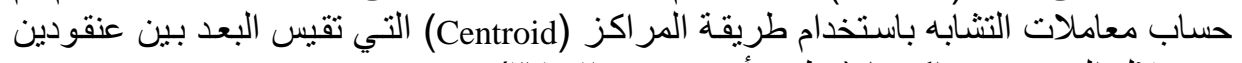

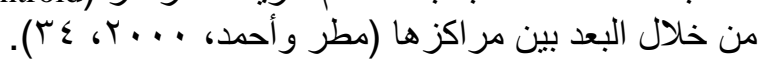




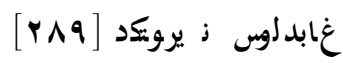

r. ب. نسبة الائتمان المحلي للقطاع الخاص إلى الناتج المحلي الإجمالي.

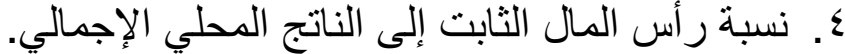

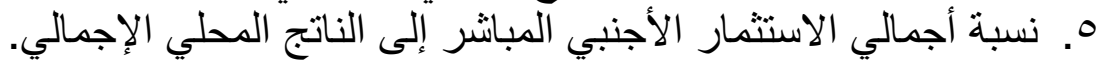

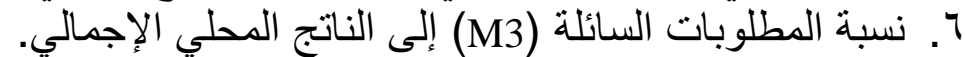

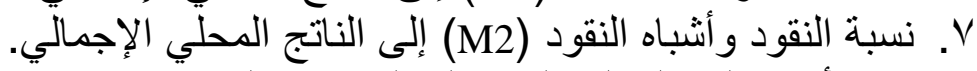

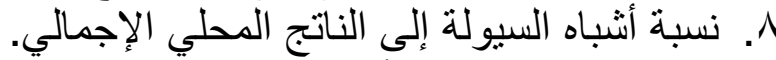
9. نسبة سرعة دوران الأسهر.

\section{Division According to Financial Development}

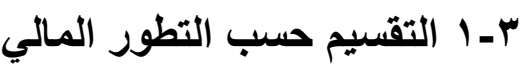

قسمت الدول حسب تطور ها المالي باستخدام عدة مؤشر ات مالية ذكرتشسابقاً

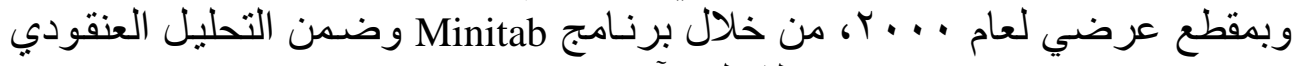
(Cluster Analysis Method)

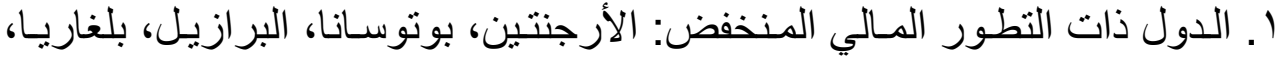

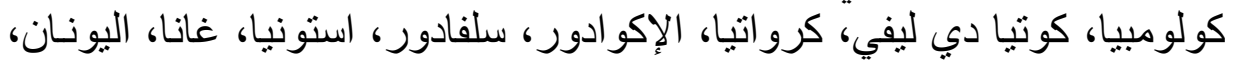

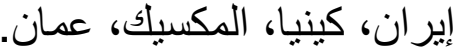
r. الدول ذات التطور المالي المتوسط: البحرين، بوليفيا، شيلي، جيك، مصر، الهند،

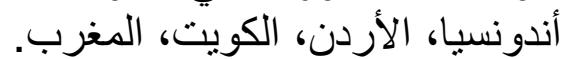

r. الدول ذات التطور المالي المرتفع: قبرص، هونك كونك، كوريا، الصين، ماليزيا،

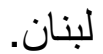

The Granger Causality Test

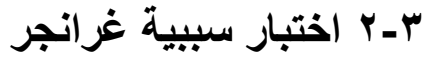

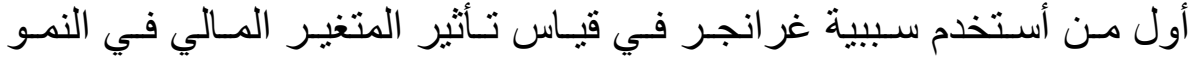

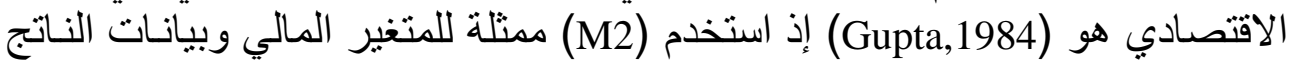

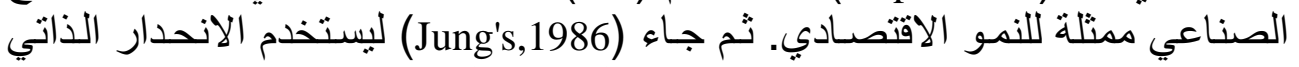

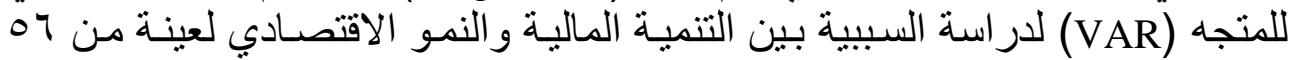

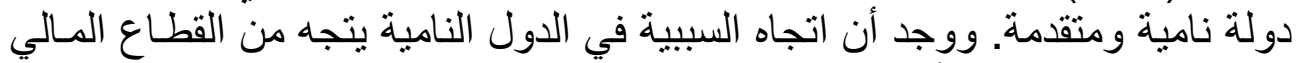

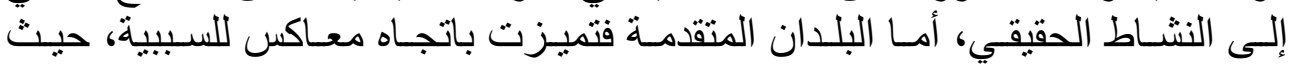

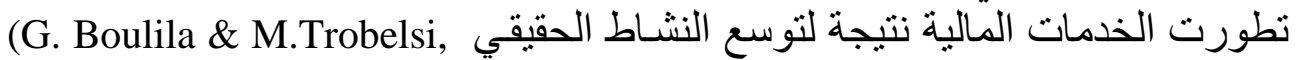

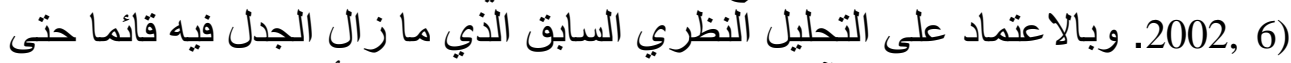

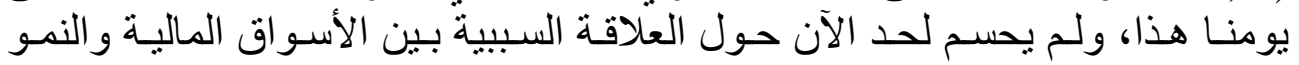

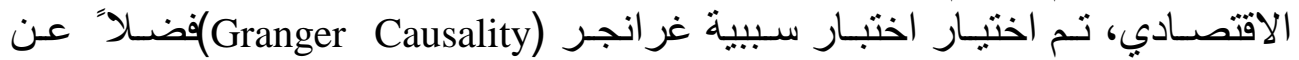

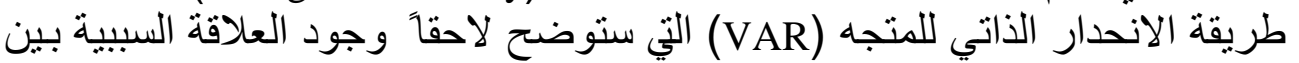

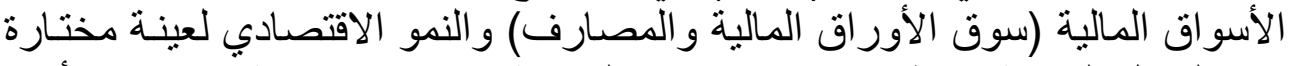

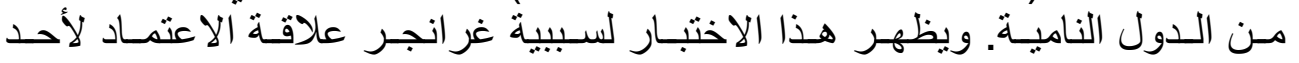

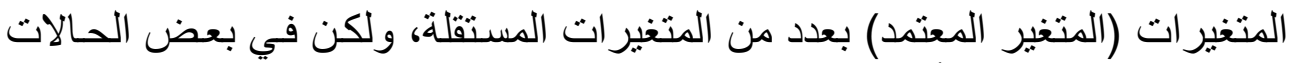

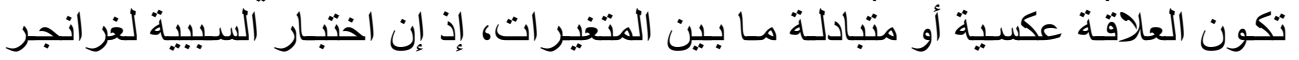


يتضمن الكثف القياسي عن اتجاه العلاقة السبيية (علاقة السبب و التأثير) عندما يكون

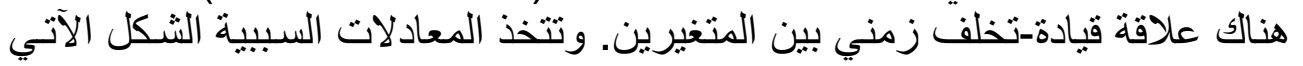
في إنموذج غرانجر (Granger, 1969, 424-438)

$$
\begin{aligned}
& \mathrm{X}_{\mathrm{t}}=\sum_{i=1}^{m} \alpha_{\mathrm{i}} \mathrm{X}_{\mathrm{t}-\mathrm{i}}+\sum_{j=1}^{m} \mathrm{~b}_{\mathrm{j}} \mathrm{Y}_{\mathrm{t}-\mathrm{j}}+\pi_{\mathrm{t}} \\
& \mathrm{Y}_{\mathrm{t}}=\sum_{i=1}^{m} \mathrm{c}_{\mathrm{i}} \mathrm{X}_{\mathrm{t}-\mathrm{i}}+\sum_{j=1}^{m} \mathrm{~d}_{\mathrm{j}} \mathrm{Y}_{\mathrm{t}-\mathrm{j}}+\mu_{\mathrm{t}}
\end{aligned}
$$

ولقد تم تحديد متغيرات البحث وتعويضها في المعادلات السابقة فتصبح بالثنكل الآتي:

$$
\begin{aligned}
& \mathrm{G}_{\mathrm{t}}=\sum_{i=1}^{m} \alpha_{\mathrm{i}} \mathrm{G}_{\mathrm{t}-\mathrm{i}}+\sum_{j=1}^{m} \mathrm{~b}_{\mathrm{j}} \mathrm{S}_{\mathrm{t}-\mathrm{j}}+\pi_{\mathrm{t}} \\
& \mathrm{S}_{\mathrm{t}}=\sum_{j=1}^{m} \mathrm{c}_{\mathrm{j}} \mathrm{S}_{\mathrm{t}-\mathrm{j}}+\sum_{i=1}^{m} \mathrm{~d}_{\mathrm{i}} \mathrm{G}_{\mathrm{t}-\mathrm{i}}+\mu_{\mathrm{t}} .
\end{aligned}
$$

$$
\begin{aligned}
& \text { علما أن X=G متغير معدل النمو. } \\
& \text { Sت S=Y } \\
& \text { = t } \\
& \text { = d ,c , b , معلمات. }
\end{aligned}
$$

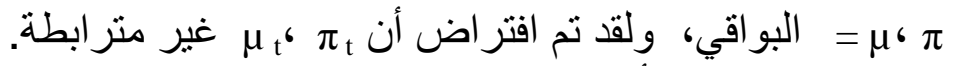

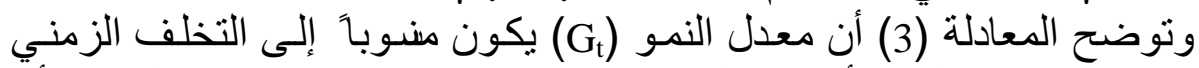

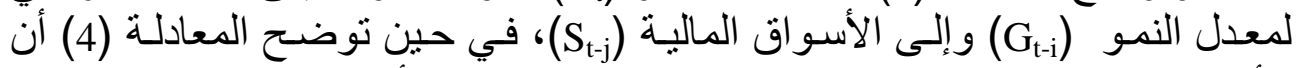

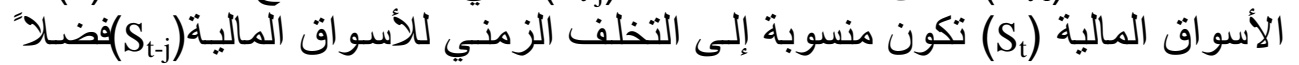

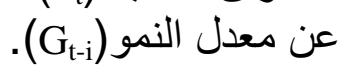

Vector Auto Regression (VAR)

r-r الانحدار الأتي للمتجهة

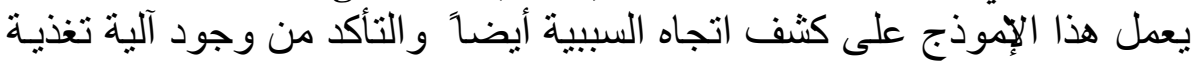

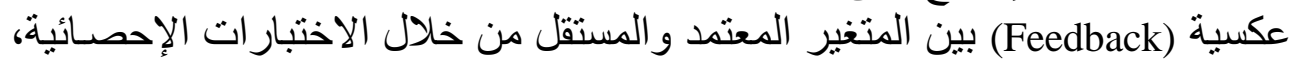

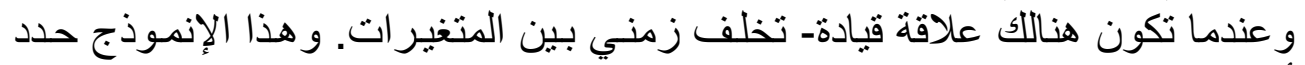

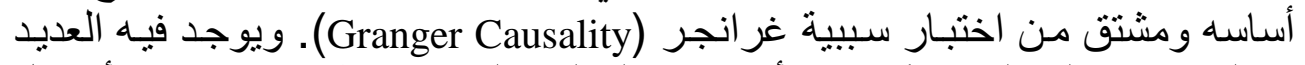

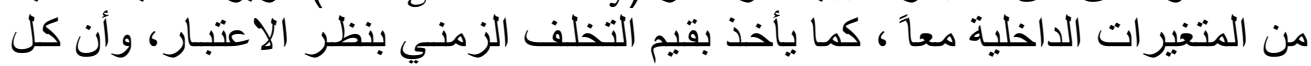

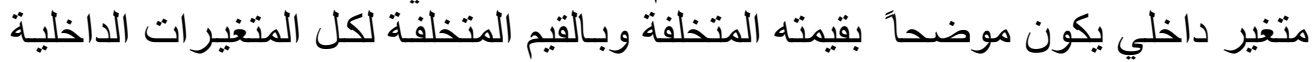

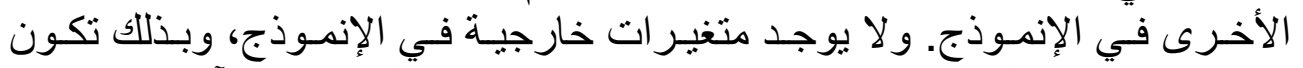

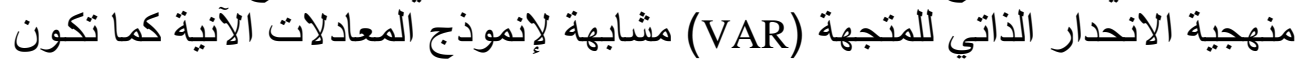

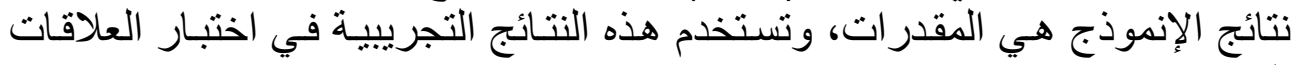
النظرية (Gujarati, 1995, 746). 


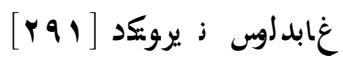

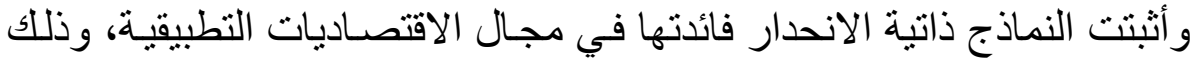

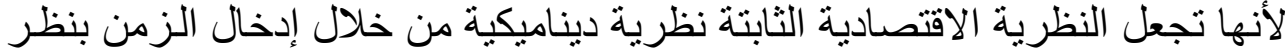

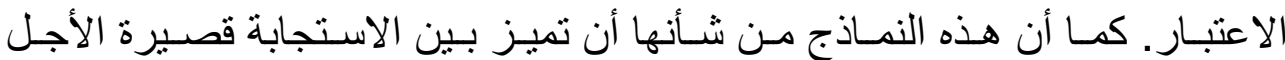

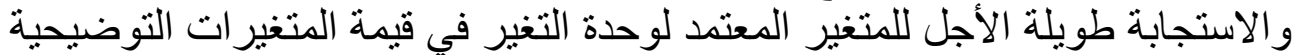

.(Gujarati, 1995, 746)

(Green, 2000, ويكون الثكل الرياضي لمعادلة الانحدار الذاتي للمتجهة هو

.740-741):

$$
\begin{aligned}
& \mathrm{Y}_{\mathrm{t}}=\mathrm{u}+\Delta_{1} \mathrm{Y}_{\mathrm{t}-1}+\ldots \ldots+\Delta_{\mathrm{p}} \mathrm{Y}_{\mathrm{t}-\mathrm{p}}+\mathrm{V}_{\mathrm{t}}
\end{aligned}
$$

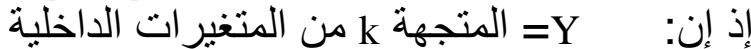

$$
\begin{aligned}
& \text { ان }=\Delta_{\mathrm{p}} \Delta_{1} \Delta_{1}
\end{aligned}
$$

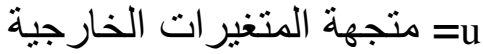

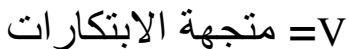

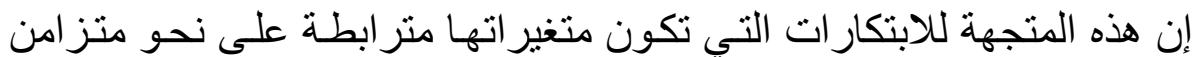

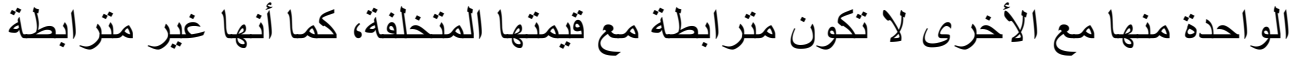

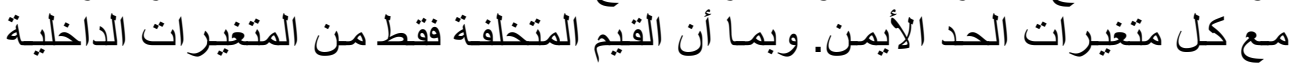

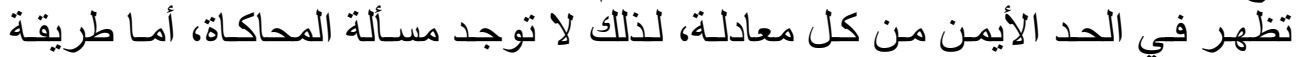

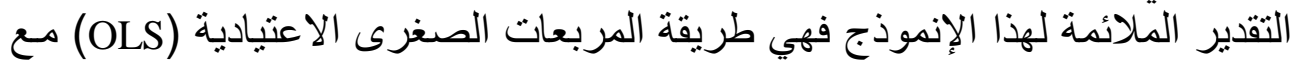

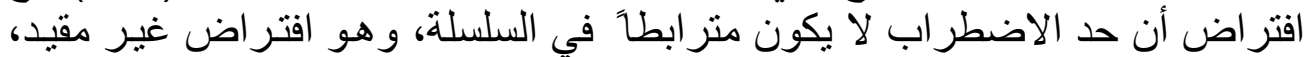

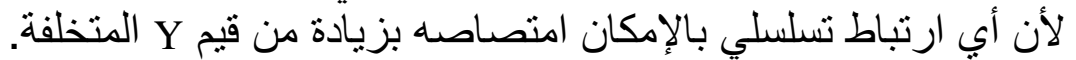

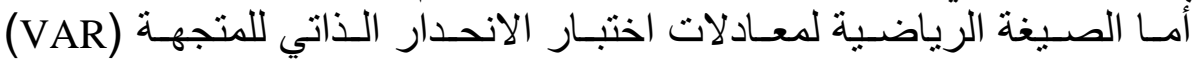

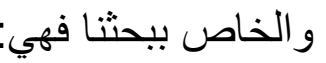

$$
\begin{aligned}
\mathrm{Y}_{\mathrm{i}, \mathrm{t}} & =\sum_{j=1}^{k} \alpha_{1 \mathrm{j}} \mathrm{Y}_{\mathrm{i}, \mathrm{t}-\mathrm{j}}+\sum_{j=1}^{k} \beta_{1 \mathrm{j}} \mathrm{B}_{\mathrm{i}, \mathrm{t}-\mathrm{j}}+\sum_{j=1}^{k} \lambda_{1 \mathrm{j}} \mathrm{S}_{\mathrm{i}, \mathrm{t}-\mathrm{j}}+\Phi_{1, \mathrm{t}}+\varepsilon_{1, \mathrm{i}, \mathrm{t}} \\
\mathrm{B}_{\mathrm{i}, \mathrm{t}} & =\sum_{j=1}^{k} \alpha_{2 \mathrm{j}} \mathrm{Y}_{\mathrm{i}, \mathrm{t}-\mathrm{j}+}+\sum_{j=1}^{k} \beta_{2 \mathrm{j}} \mathrm{B}_{\mathrm{i}, \mathrm{t}-\mathrm{j}}+\sum_{j=1}^{k} \lambda_{2 \mathrm{j}} \mathrm{S}_{\mathrm{i}, \mathrm{t}-\mathrm{j}}+\Phi_{2, \mathrm{t}}+\varepsilon_{2, \mathrm{i}, \mathrm{t}} . \\
\mathrm{S}_{\mathrm{i}, \mathrm{t}} & =\sum_{j=1}^{k} \alpha_{3 \mathrm{j}} \mathrm{Y}_{\mathrm{i}, \mathrm{t}-\mathrm{j}}+\sum_{j=1}^{k} \beta_{3 \mathrm{j}} \mathrm{B}_{\mathrm{i}, \mathrm{t}-\mathrm{j}}+\sum_{j=1}^{k} \lambda_{3 \mathrm{j}} \mathrm{S}_{\mathrm{i}, \mathrm{t} \mathrm{j}+\Phi_{3, \mathrm{t}}+\varepsilon_{3, \mathrm{i}, \mathrm{t}}}
\end{aligned}
$$

$$
\begin{aligned}
& \text { إذ إن: = = متغير النمو الاقتصادي. }
\end{aligned}
$$

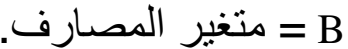

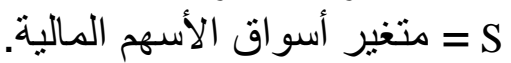

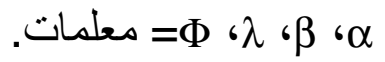

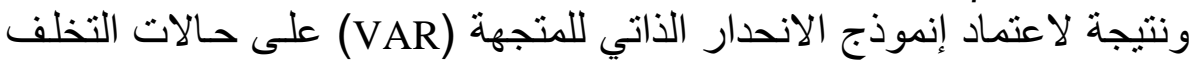

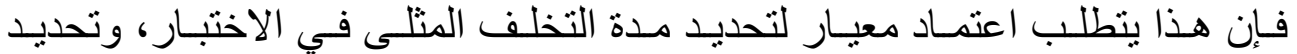

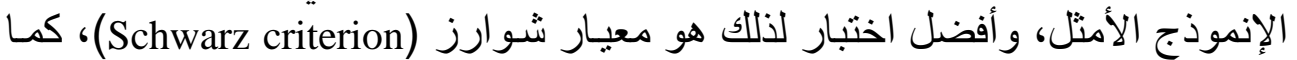

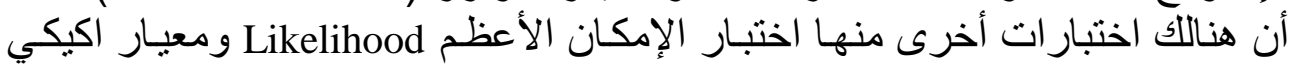

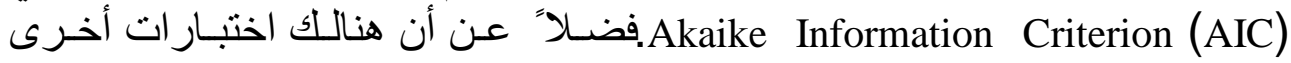




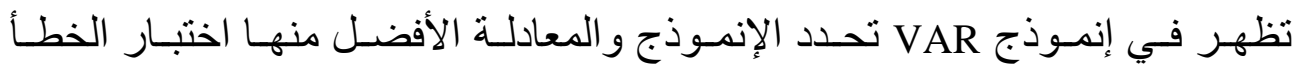

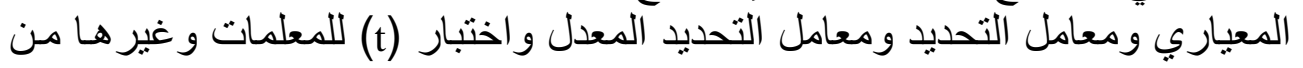
الاختبار أت الإحصائية المعروفئة لقد تم استخدام متغيرين بمثلان المصائنة المصارف في المعادلات المقات المقرة وهما:

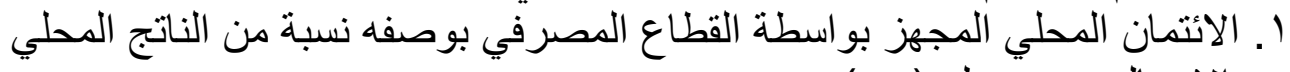

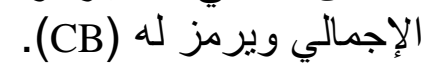

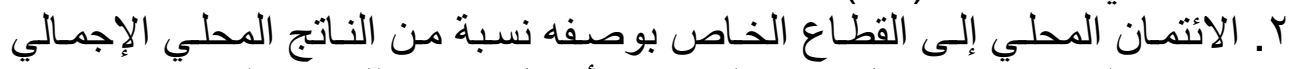

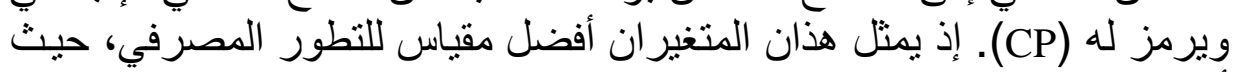

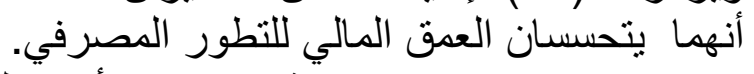

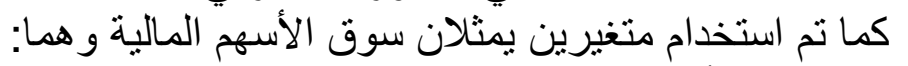

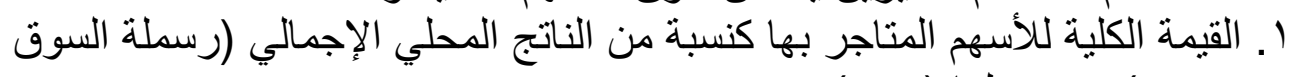

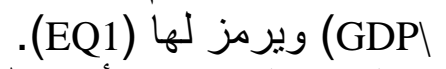
r. نسبة سرعة دوران الأسهم المتاجر بها ويرمز لهان لها (EQ2).

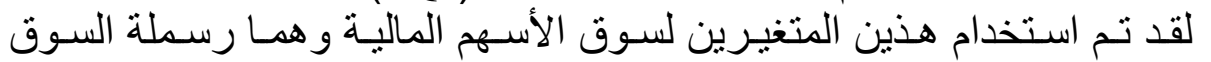

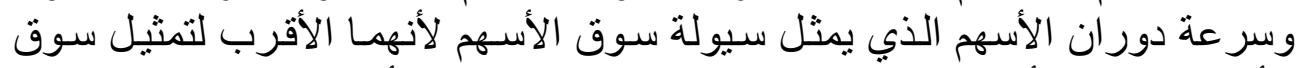

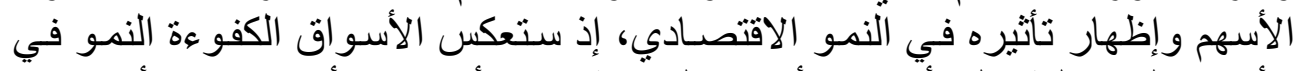

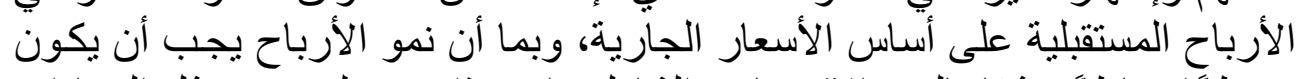

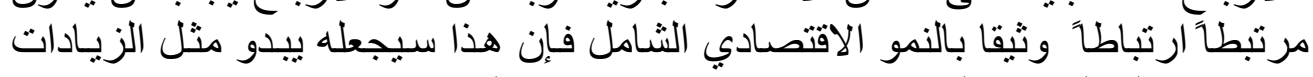

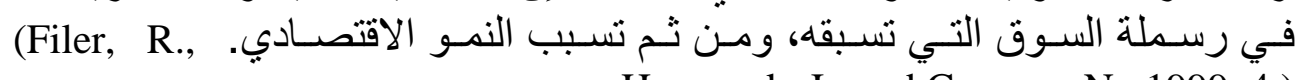
Hanousek, J., and Campos, N., 1999 ,4

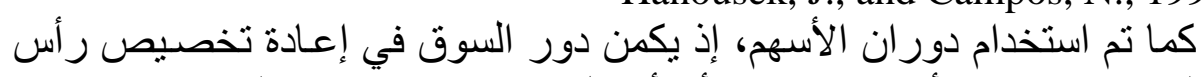

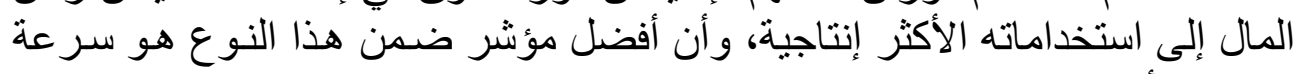

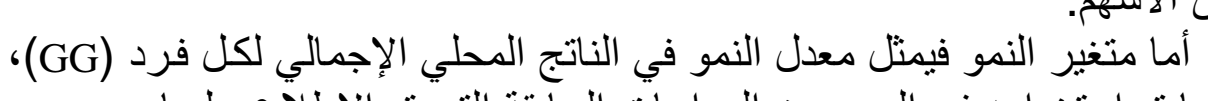

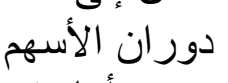

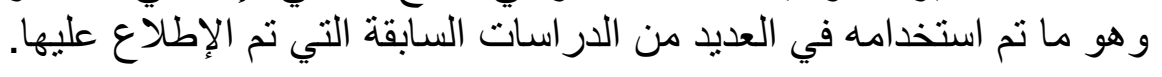

ع. النتائج التجريبية

\section{Lower-Financial Development States}

ع ـ الأجول ذاتجبية التطور المالي المنخفض

الجدول ا يمثل سببية غرانجر للدول ذات التطور المـالي المنخفض. ويظهر

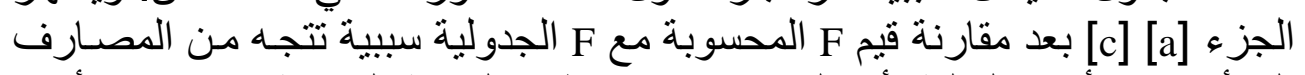

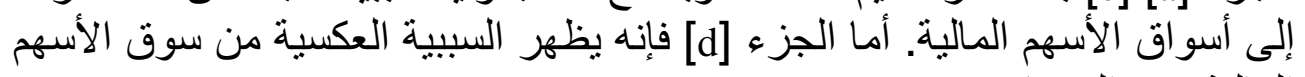
المالية نحو المصارف الاسف.

\section{الجدول الت الت}

اختبار سبيية غرانجر للاول ذات التطور المالي المخفض 


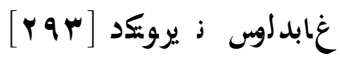

[a]

\begin{tabular}{|l|c|c|}
\hline Lags: 18 & \multicolumn{3}{|l|}{} \\
\hline & F-Statistic & Probability \\
\hline $\mathrm{EQ} 1 \rightarrow \mathrm{CB}$ & 0.55319 & 0.90356 \\
\hline $\mathrm{CB} \rightarrow \mathrm{EQ} 1$ & $6.85763^{* *}$ & $3.9 \mathrm{E}-06$ \\
\hline $\mathrm{GG} \rightarrow \mathrm{CB}$ & 1.44199 & 0.13055 \\
\hline $\mathrm{CB} \rightarrow \mathrm{GG}$ & 0.52239 & 0.94108 \\
\hline $\mathrm{GG} \rightarrow \mathrm{EQ} 1$ & 0.94002 & 0.53708 \\
\hline $\mathrm{EQ} 1 \rightarrow \mathrm{GG}$ & 0.97030 & 0.50457 \\
\hline
\end{tabular}

[d]

\begin{tabular}{|l|c|c|}
\hline Lags: 21 & \multicolumn{3}{|c|}{} \\
\hline & F-Statistic & Probability \\
\hline $\mathrm{EQ} 1 \rightarrow \mathrm{CP}$ & 1.12306 & 0.46874 \\
\hline $\mathrm{CP} \rightarrow \mathrm{EQ} 1$ & $2.00931^{*}$ & 0.17437 \\
\hline $\mathrm{GG} \rightarrow \mathrm{CP}$ & 0.35767 & 0.99509 \\
\hline $\mathrm{CP} \rightarrow \mathrm{GG}$ & 0.80162 & 0.70953 \\
\hline $\mathrm{GG} \rightarrow \mathrm{EQ} 1$ & 0.86437 & 0.63162 \\
\hline $\mathrm{EQ} 1 \rightarrow \mathrm{GG}$ & 1.07106 & 0.41416 \\
\hline
\end{tabular}

[b]

\begin{tabular}{|l|c|c|}
\hline Lags: 5 & \\
\hline & F-Statistic & Probability \\
\hline $\mathrm{EQ} 2 \rightarrow \mathrm{CB}$ & $2.48510^{*}$ & 0.03933 \\
\hline $\mathrm{CB} \rightarrow \mathrm{EQ} 2$ & $2.85188^{*}$ & 0.02104 \\
\hline $\mathrm{GG} \rightarrow \mathrm{CB}$ & 0.70426 & 0.62103 \\
\hline $\mathrm{CB} \rightarrow \mathrm{GG}$ & 1.34294 & 0.24895 \\
\hline $\mathrm{GG} \rightarrow \mathrm{EQ} 2$ & 1.11506 & 0.35889 \\
\hline $\mathrm{EQ} 2 \rightarrow \mathrm{GG}$ & 0.48154 & 0.78909 \\
\hline
\end{tabular}

[c]

\begin{tabular}{|c|c|c|}
\hline \multicolumn{3}{|l|}{ ags: 14} \\
\hline & F-Statistic & Probability \\
\hline$\overline{\mathrm{EQ} 2} \rightarrow \mathrm{CP}$ & $2.91922 *$ & 0.28426 \\
\hline $\mathrm{CP} \rightarrow \mathrm{EQ} 2$ & 0.61061 & 0.77044 \\
\hline $\mathrm{GG} \rightarrow \mathrm{CP}$ & 0.44440 & 0.95624 \\
\hline $\mathrm{CP} \rightarrow \mathrm{GG}$ & 1.23963 & 0.25698 \\
\hline $\mathrm{GG} \rightarrow \mathrm{EQ} 2$ & 0.66720 & 0.77870 \\
\hline $\mathrm{EQ} 2 \rightarrow \mathrm{GG}$ & 1.42947 & 0.22687 \\
\hline
\end{tabular}

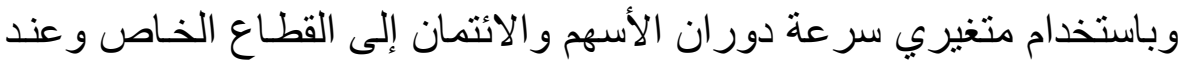

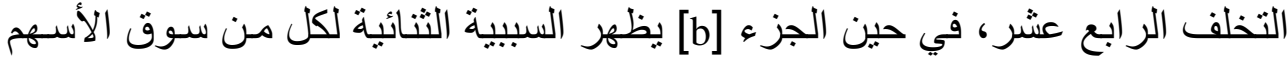

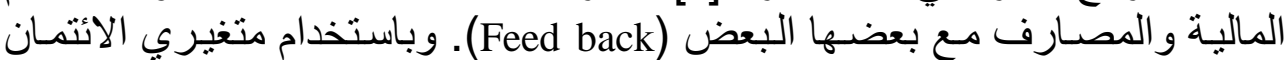

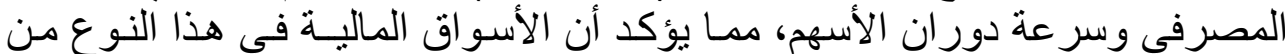

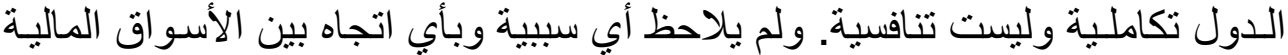

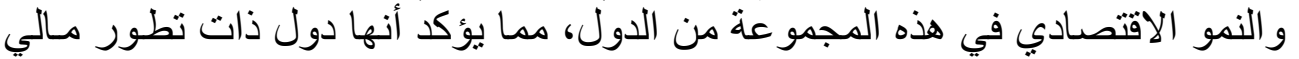

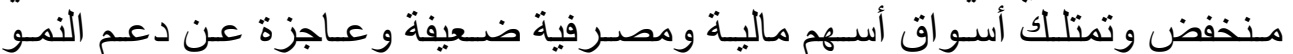
الاقتصادي فيها.

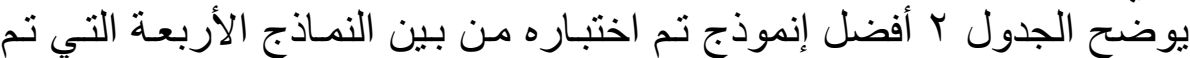

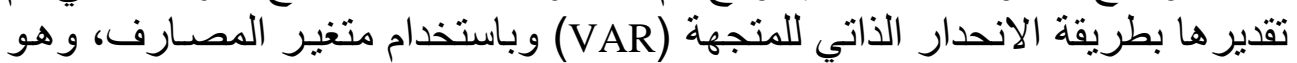
الائتمان المحلي إلى القطاع الخـاص كنسبة من GDP ومتغير سوق الأسـهم الماليـة

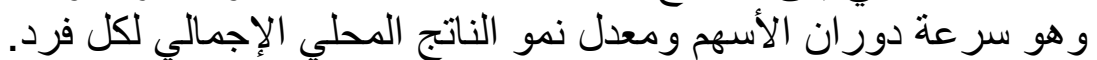

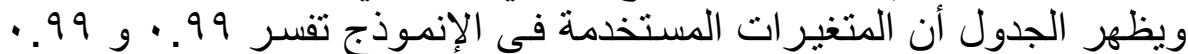

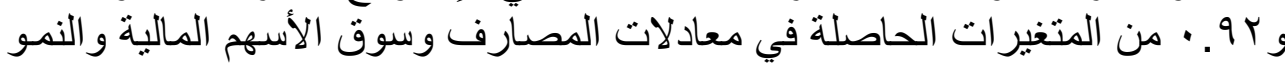

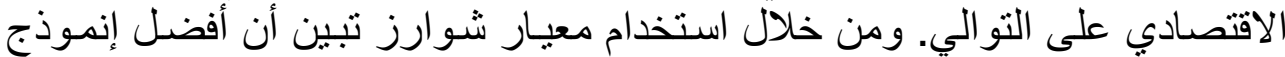

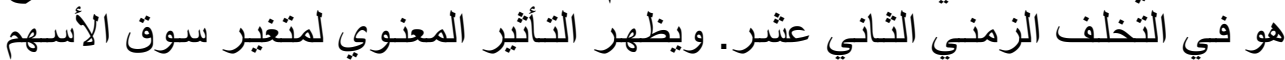

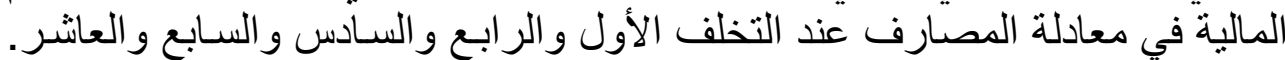

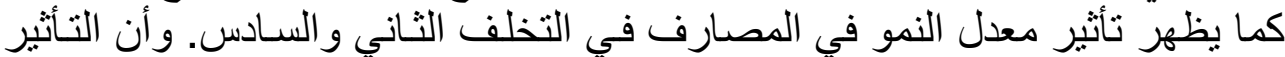

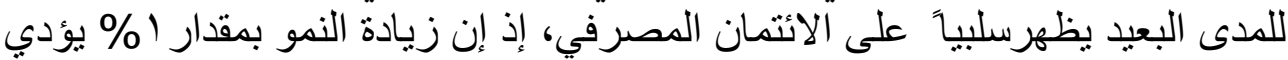

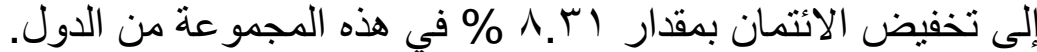




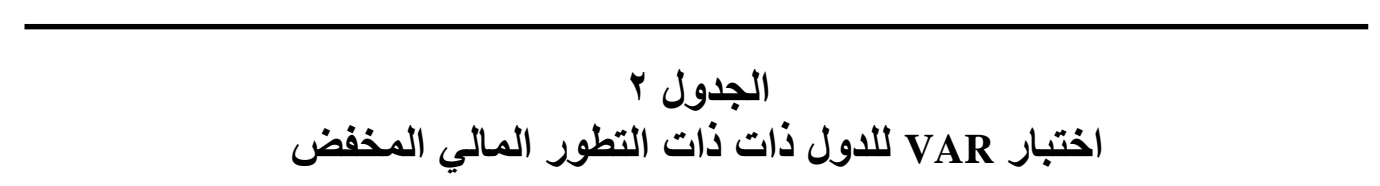

\begin{tabular}{|c|c|c|c|}
\hline & CP & EQ2 & GG \\
\hline $\mathrm{CP}(-1)$ & 4.936168 & 0.947211 & -0.508834 \\
\hline & $(3.04638)$ & $(2.58597)$ & $(1.98831)$ \\
\hline & $(1.62034)$ & $(0.36629)$ & $(-0.25591)$ \\
\hline $\mathrm{CP}(-2)$ & -0.146139 & -6.542476 & 0.956150 \\
\hline & $(2.65223)$ & $(2.25140)$ & $(1.73106)$ \\
\hline & $(-0.05510)$ & $(-2.90596)^{\star}$ & $(0.55235)$ \\
\hline $\mathrm{CP}(-3)$ & -6.080574 & 8.649441 & -0.954875 \\
\hline & $(2.49483)$ & $(2.11778)$ & $(1.62833)$ \\
\hline & $(-2.43727)^{\star}$ & $(4.08421)^{\star *}$ & $(-0.58642)$ \\
\hline $\mathrm{CP}(-4)$ & 3.861825 & -5.285473 & 0.590745 \\
\hline & $(1.47903)$ & $(1.25550)$ & $(0.96533)$ \\
\hline & $(2.61106)^{\star}$ & $(-4.20986)^{\star *}$ & $(0.61196)$ \\
\hline $\mathrm{CP}(-5)$ & -3.790237 & 3.367503 & -0.201402 \\
\hline & $(1.82216)$ & $(1.54677)$ & $(1.18929)$ \\
\hline & $(-2.08008)^{\star}$ & $(2.17712)^{\star}$ & $(-0.16935)$ \\
\hline $\mathrm{CP}(-6)$ & -0.431600 & 0.165896 & 0.189206 \\
\hline & $(0.42259)$ & $(0.35872)$ & $(0.27581)$ \\
\hline & $(-1.02133)$ & $(0.46246)$ & $(0.68599)$ \\
\hline $\mathrm{CP}(-7)$ & 3.654863 & -1.194193 & -0.084067 \\
\hline & $(2.02729)$ & $(1.72090)$ & $(1.32317)$ \\
\hline & $(1.80283)^{*}$ & $(-0.69393)$ & $(-0.06353)$ \\
\hline $\mathrm{CP}(-8)$ & 0.814164 & -1.297251 & 0.007055 \\
\hline & $(0.58331)$ & $(0.49515)$ & $(0.38071)$ \\
\hline & $(1.39578)$ & $(-2.61992)^{\star}$ & $(0.01853)$ \\
\hline $\mathrm{CP}(-9)$ & -0.873652 & 1.105030 & -0.054970 \\
\hline & $(0.56793)$ & $(0.48210)$ & $(0.37068)$ \\
\hline & $(-1.53830)$ & $(2.29212)^{*}$ & $(-0.14829)$ \\
\hline $\mathrm{CP}(-10)$ & 2.430299 & -2.247646 & 0.002935 \\
\hline & $(1.22047)$ & $(1.03601)$ & $(0.79658)$ \\
\hline & $(1.99129)^{\star}$ & $(-2.16951)^{\star}$ & $(0.00368)$ \\
\hline $\mathrm{CP}(-11)$ & -2.575393 & 0.816951 & -0.084598 \\
\hline & $(1.43263)$ & $(1.21611)$ & $(0.93505)$ \\
\hline & $(-1.79767)$ & $(0.67177)$ & $(-0.09048)$ \\
\hline $\mathrm{CP}(-12)$ & -2.576818 & 1.727605 & -0.017385 \\
\hline & $(1.34473)$ & $(1.14150)$ & $(0.87768)$ \\
\hline & $(-1.91623)^{\star}$ & $(1.51345)$ & $(-0.01981)$ \\
\hline & -1.901593 & 3.429138 & -0.384650 \\
\hline & $(0.91640)$ & $(0.77791)$ & $(0.59812)$ \\
\hline & $(-2.07506)^{\star}$ & $(4.40816)^{\star \star}$ & $(-0.64310)$ \\
\hline & & \\
\hline & & & \\
\hline & &
\end{tabular}

\begin{tabular}{|c|c|c|c|}
\hline & CP & EQ2 & GG \\
\hline & $(1.05689)$ & $(0.89716)$ & $(0.68981)$ \\
\hline & $(0.47416)$ & $(-2.07995)^{\star}$ & $(0.71307)$ \\
\hline EQ2(-3) & 0.414263 & 0.646658 & -0.037216 \\
\hline & $(0.41999)$ & $(0.35651)$ & $(0.27412)$ \\
\hline & $(0.98637)$ & $(1.81383)$ & $(-0.13577)$ \\
\hline EQ2(-4) & 1.113850 & -1.248651 & 0.135827 \\
\hline & $(0.57297)$ & $(0.48637)$ & $(0.37396)$ \\
\hline & $(1.94401)^{\star}$ & $(-2.56727)^{\star}$ & $(0.36321)$ \\
\hline EQ2(-5) & -0.722472 & 0.837682 & -0.084722 \\
\hline & $(0.44355)$ & $(0.37652)$ & $(0.28950)$ \\
\hline & $(-1.62883)$ & $(2.22482)^{\star}$ & $(-0.29265)$ \\
\hline EQ2(-6) & -0.490770 & 0.373448 & 0.026517 \\
\hline & $(0.24963)$ & $(0.21190)$ & $(0.16293)$ \\
\hline & $(-1.96598)^{\star}$ & $(1.76234)$ & $(0.16275)$ \\
\hline EQ2(-7) & -1.150117 & 1.619585 & -0.259178 \\
\hline & $(0.57690)$ & $(0.48971)$ & $(0.37653)$ \\
\hline & $(-1.99363)^{\star}$ & $(3.30725)^{\star *}$ & $(-0.68833)$ \\
\hline EQ2(-8) & 0.383034 & -0.489532 & 0.305339 \\
\hline & $(0.55234)$ & $(0.46886)$ & $(0.36050)$ \\
\hline & $(0.69347)$ & $(-1.04408)$ & $(0.84698)$ \\
\hline EQ2(-9) & 0.927872 & -0.818356 & 0.243428 \\
\hline & $(0.64340)$ & $(0.54616)$ & $(0.41993)$ \\
\hline & $(1.44215)$ & $(-1.49839)$ & $(0.57968)$ \\
\hline EQ2(-10) & -0.571388 & 0.449091 & -0.165274 \\
\hline & $(0.31303)$ & $(0.26572)$ & $(0.20431)$ \\
\hline & $(-1.82537)^{\star}$ & $(1.69010)$ & $(-0.80895)$ \\
\hline EQ2(-11) & -0.276533 & 0.319754 & -0.048264 \\
\hline & $(0.30716)$ & $(0.26074)$ & $(0.20048)$ \\
\hline & $(-0.90030)$ & $(1.22636)$ & $(-0.24075)$ \\
\hline EQ2(-12) & 0.276227 & -0.750613 & -0.014881 \\
\hline & $(0.25914)$ & $(0.21997)$ & $(0.16913)$ \\
\hline & $(1.06594)$ & $(-3.41227)^{\star *}$ & $(-0.08798)$ \\
\hline GG(-1) & 1.932986 & -10.30863 & 1.499470 \\
\hline & $(3.82298)$ & $(3.24521)$ & $(2.49519)$ \\
\hline & $(0.50562)$ & $(-3.17657)^{* *}$ & $(0.60094)$ \\
\hline GG(-2) & -5.998568 & 6.205206 & -0.583229 \\
\hline & $(2.36338)$ & $(2.00620)$ & $(1.54253)$ \\
\hline & $(-2.53813)^{\star}$ & $(3.09302)^{\star *}$ & $(-0.37810)$ \\
\hline & & & \\
\hline & & & \\
\hline & & & \\
& &
\end{tabular}




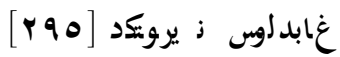

\begin{tabular}{|l|r|r|r|}
\hline & \multicolumn{1}{|c|}{ CP } & \multicolumn{1}{c|}{ EQ2 } & \multicolumn{1}{c|}{ GG } \\
\hline GG(-10) & -3.022583 & -0.597514 & 0.450584 \\
\hline & $(3.01792)$ & $(2.56182)$ & $(1.96974)$ \\
\hline & $(-1.00154)$ & $(-0.23324)$ & $(0.22875)$ \\
\hline GG(-11) & -4.295528 & 4.059349 & -0.013199 \\
\hline & $(2.60737)$ & $(2.21331)$ & $(1.70178)$ \\
\hline & $(-1.64746)$ & $(1.83406)$ & $(-0.00776)$ \\
\hline GG(-12) & 0.017283 & -0.194281 & 1.254707 \\
\hline & $(2.01706)$ & $(1.71221)$ & $(1.31649)$ \\
\hline & $(0.00857)$ & $(-0.11347)$ & $(0.95307)$ \\
\hline \multicolumn{1}{|c|}{$\mathrm{C}$} & 88.76895 & -53.97813 & -2.904786 \\
\hline & $(46.4216)$ & $(39.4058)$ & $(30.2985)$ \\
\hline & $(1.91223)$ & $(-1.36980)$ & $(-0.09587)$ \\
\hline $\mathrm{R}^{2}-$ & 0.992384 & 0.996780 & 0.929640 \\
\hline Adj. $\mathrm{R}^{-2}$ & 0.718214 & 0.880867 & -1.603319 \\
\hline S.E. & 8.667717 & 7.357746 & 5.657255 \\
\hline F- & 3.619589 & 8.599333 & 0.367017 \\
\hline $\begin{array}{l}\text { Log } \\
\text { likelihood }\end{array}$ & -66.87053 & -60.64416 & -50.65720 \\
\hline AIC & 5.466870 & 5.139166 & 4.613537 \\
\hline SC & 7.061362 & 6.733658 & 6.208028 \\
\hline *, **, Indicate significance at the 5\%, 1\% level
\end{tabular}

\begin{tabular}{|l|c|c|c|}
\hline & CP & EQ2 & GG \\
\hline GG(-10) & -3.022583 & -0.597514 & 0.450584 \\
\hline & $(3.01792)$ & $(2.56182)$ & $(1.96974)$ \\
\hline & $(-1.00154)$ & $(-0.23324)$ & $(0.22875)$ \\
\hline GG(-11) & -4.295528 & 4.059349 & -0.013199 \\
\hline & $(2.60737)$ & $(2.21331)$ & $(1.70178)$ \\
\hline & $(-1.64746)$ & $(1.83406)$ & $(-0.00776)$ \\
\hline GG(-12) & 0.017283 & -0.194281 & 1.254707 \\
\hline & $(2.01706)$ & $(1.71221)$ & $(1.31649)$ \\
\hline & $(0.00857)$ & $(-0.11347)$ & $(0.95307)$ \\
\hline \multicolumn{1}{|c|}{$\mathrm{C}$} & 88.76895 & -53.97813 & -2.904786 \\
\hline & $(46.4216)$ & $(39.4058)$ & $(30.2985)$ \\
\hline & $(1.91223)$ & $(-1.36980)$ & $(-0.09587)$ \\
\hline$R^{2}-$ & 0.992384 & 0.996780 & 0.929640 \\
\hline Adj. $\mathrm{R}^{-2}$ & 0.718214 & 0.880867 & -1.603319 \\
\hline S.E. & 8.667717 & 7.357746 & 5.657255 \\
\hline F- & 3.619589 & 8.599333 & 0.367017 \\
\hline $\begin{array}{l}\text { Log } \\
\text { likelihood }\end{array}$ & -66.87053 & -60.64416 & -50.65720 \\
\hline AIC & 5.466870 & 5.139166 & 4.613537 \\
\hline SC & 7.061362 & 6.733658 & 6.208028 \\
\hline *, **, Indicate significance at the 5\%,1\% level
\end{tabular}

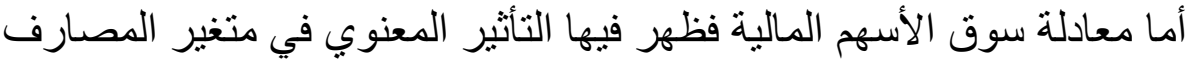

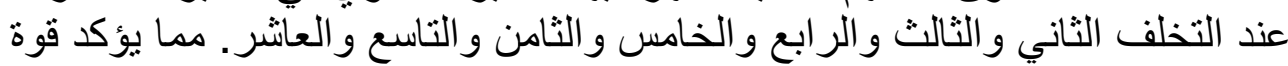

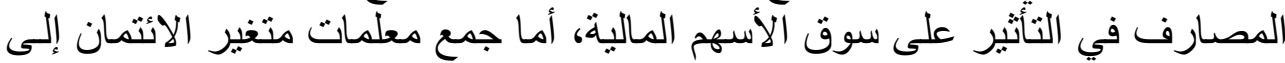

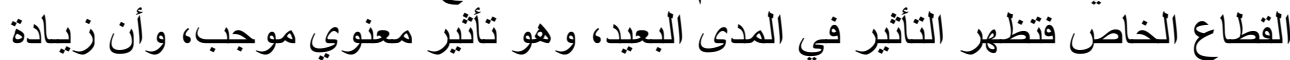

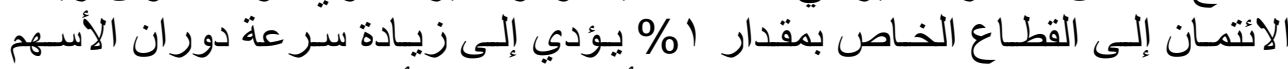

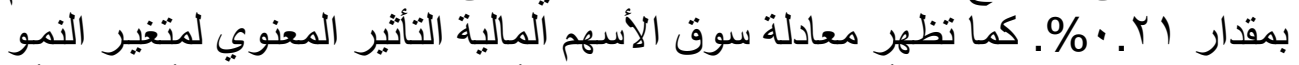

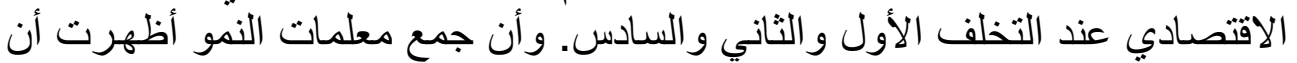

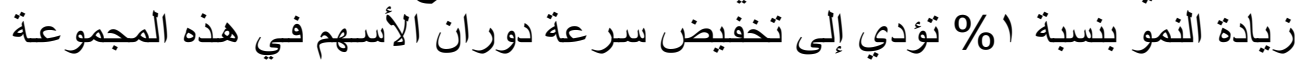

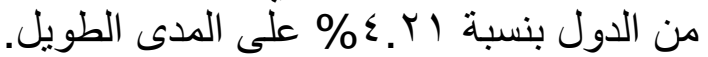

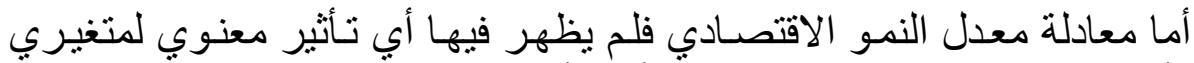

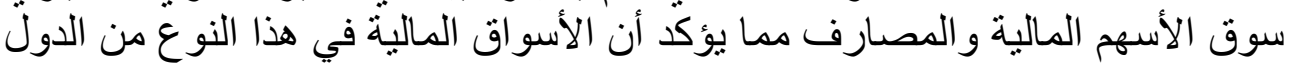

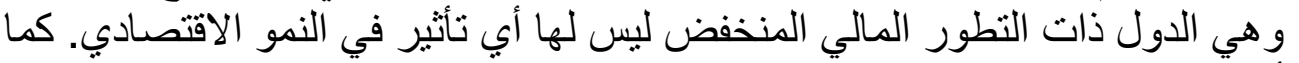

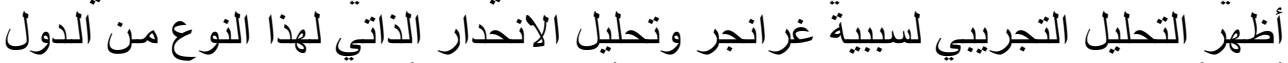

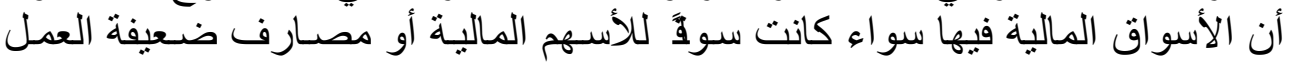

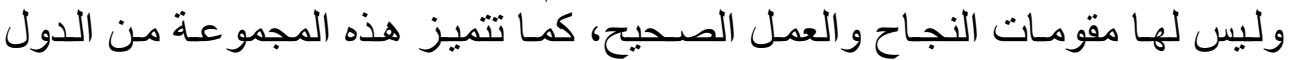

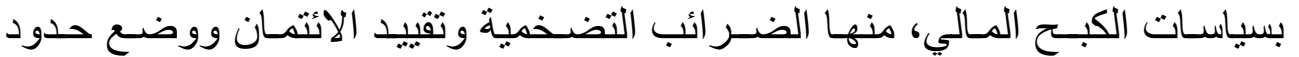

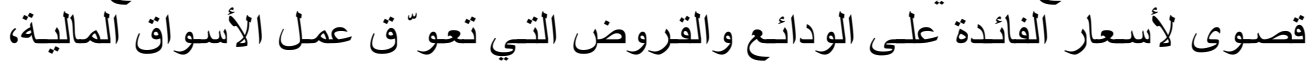

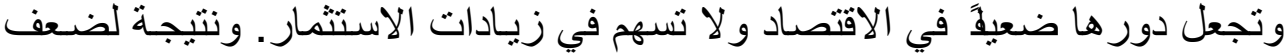

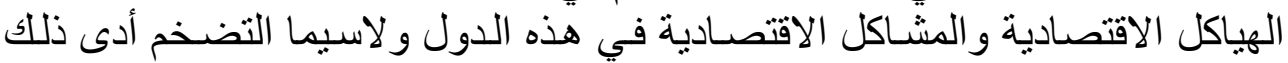

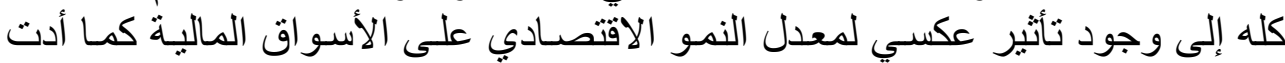

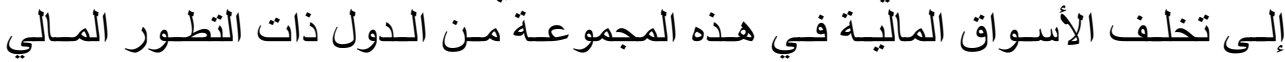




\section{Middle-Financial Development States}

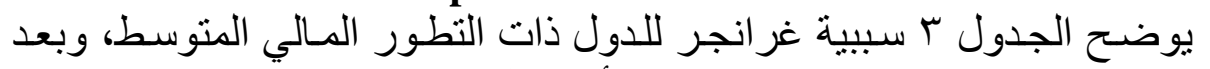

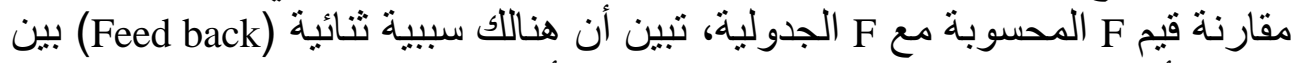

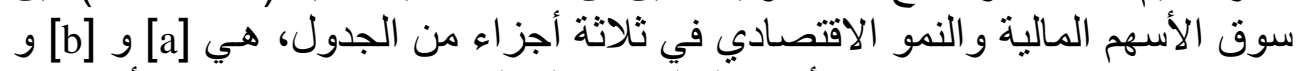

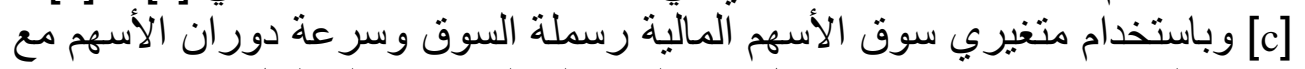

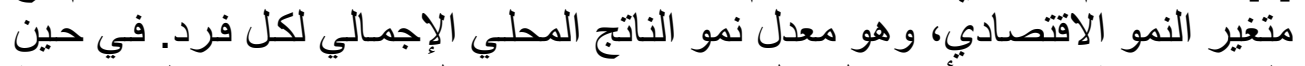

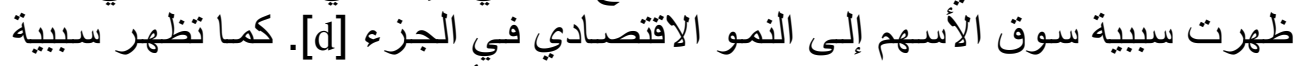

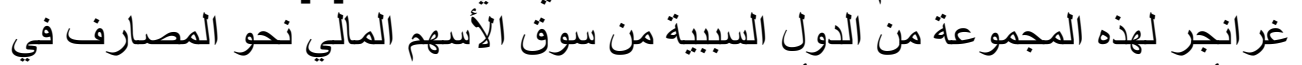

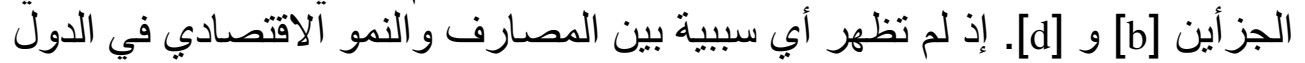
ذات التطور المالي المتوسط.

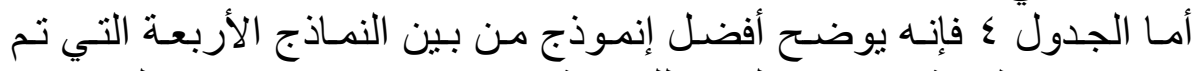

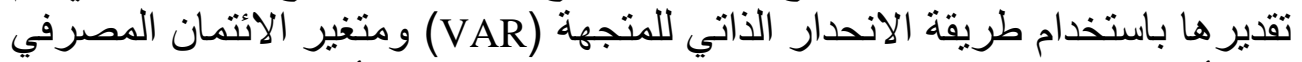

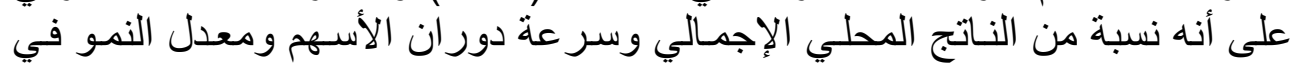

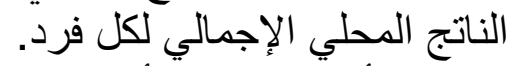

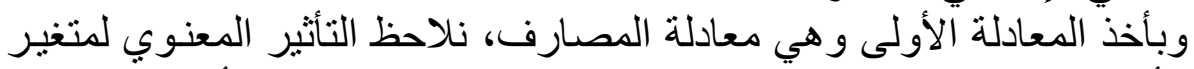

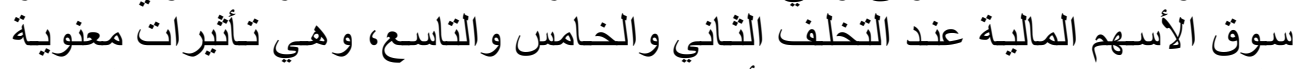

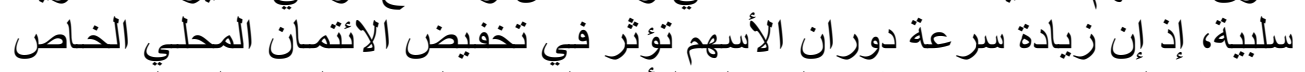

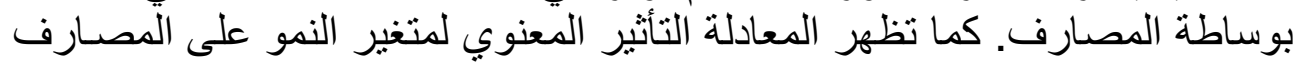
عند التخلف الثالث و الر ابع و السادس و التاسع.

\section{الجدول ب البط}

[a]

\begin{tabular}{|l|c|c|}
\hline \multicolumn{2}{|l|}{ Lags: 6} \\
\hline EQ1 $\rightarrow$ CB & F-Statistic & Probability \\
\hline $\mathrm{CB} \rightarrow \mathrm{EQ} 1$ & 1.13286 & 0.05802 \\
\hline $\mathrm{GG} \rightarrow \mathrm{CB}$ & 2.02854 & 0.41274 \\
\hline $\mathrm{CB} \rightarrow \mathrm{GG}$ & 0.86789 & 0.05824 \\
\hline $\mathrm{GG} \rightarrow \mathrm{EQ1}$ & $2.98665^{*}$ & 0.52147 \\
\hline $\mathrm{EQ} 1 \rightarrow \mathrm{GG}$ & $2.62740^{*}$ & 0.01084 \\
\hline
\end{tabular}

[c]

\begin{tabular}{|l|c|c|}
\hline \multicolumn{3}{|l|}{ Lags: 6} \\
\hline & F-Statistic & Probability \\
\hline EQ1 $\rightarrow$ CP & 0.72388 & 0.63154 \\
\hline $\mathrm{CP} \rightarrow \mathrm{EQ} 1$ & 2.11036 & 0.06058 \\
\hline $\mathrm{GG} \rightarrow \mathrm{CP}$ & 1.72573 & 0.12306 \\
\hline $\mathrm{CP} \rightarrow \mathrm{GG}$ & 1.26606 & 0.28019 \\
\hline $\mathrm{GG} \rightarrow \mathrm{EQ} 1$ & $2.98665^{\star}$ & 0.01084 \\
\hline $\mathrm{EQ} 1 \rightarrow \mathrm{GG}$ & $2.62740^{*}$ & 0.02209 \\
\hline
\end{tabular}

[b]

\begin{tabular}{|c|c|c|}
\hline \multicolumn{3}{|l|}{ Lags: 14} \\
\hline & F-Statistic & Probability \\
\hline $\mathrm{EQ} 2 \rightarrow \mathrm{CB}$ & $15.6505^{\star *}$ & 0.00838 \\
\hline $\mathrm{CB} \rightarrow \mathrm{EQ} 2$ & 0.88246 & 0.61927 \\
\hline $\mathrm{GG} \rightarrow \mathrm{CB}$ & $2.06820^{*}$ & 0.02526 \\
\hline $\mathrm{CB} \rightarrow \mathrm{GG}$ & 0.95830 & 0.50416 \\
\hline $\mathrm{GG} \rightarrow \mathrm{EQ} 2$ & $4.64546^{* *}$ & 0.07444 \\
\hline EQ2 $\rightarrow$ GG & $3.33923^{* *}$ & 0.12656 \\
\hline
\end{tabular}

[d]

\begin{tabular}{l|c|c|}
\hline Lags: 12 & \\
\hline & F-Statistic & Probability \\
\hline EQ2 $\rightarrow$ CP & $5.00228^{\star *}$ & 0.00279 \\
\hline $\mathrm{CP} \rightarrow \mathrm{EQ} 2$ & 0.51174 & 0.87424 \\
\hline $\mathrm{GG} \rightarrow \mathrm{CP}$ & 1.29440 & 0.24049 \\
\hline $\mathrm{CP} \rightarrow \mathrm{GG}$ & 1.04411 & 0.41933 \\
\hline $\mathrm{GG} \rightarrow \mathrm{EQ} 2$ & 0.90429 & 0.56463 \\
\hline $\mathrm{EQ} 2 \rightarrow \mathrm{GG}$ & $2.47631^{\star}$ & 0.05423 \\
\hline
\end{tabular}

$*, * *$, indicate significant at 5\%,1\% level 


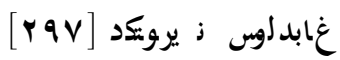

أمـا معادلـة النمـو فإنهـا أفضـل معادلـة في الإنمـوذج وحسـب معيـار الإمكـان

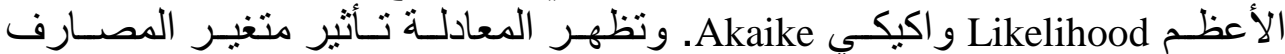

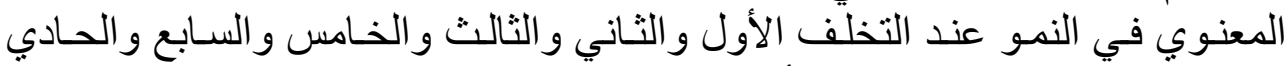
عشر و الثاني عشر ـ مما يؤكد التأثثر القوي للمصارف في في هذه المجمو عـة من الدول

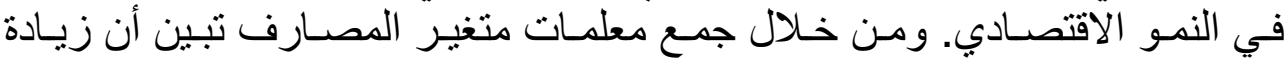

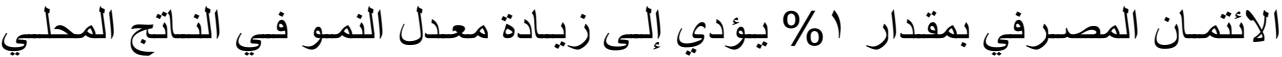

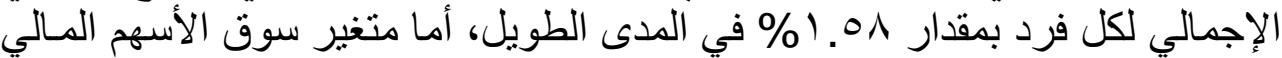

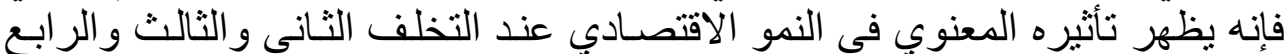

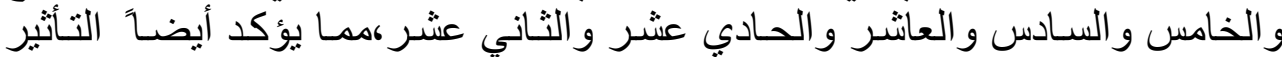

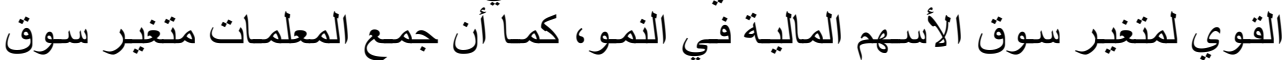

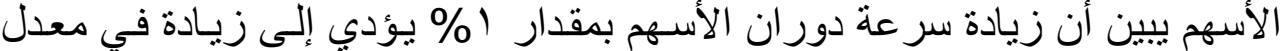

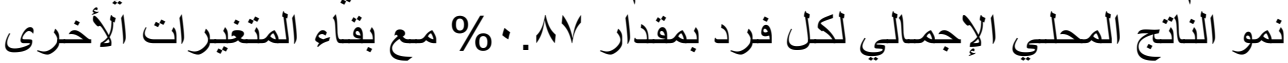
ثنابتة.

مدـا سبتق يلاحظظ أن الأسـواق الماليـة وهمـا المصـارف وسـوق الأسـهم الماليـة

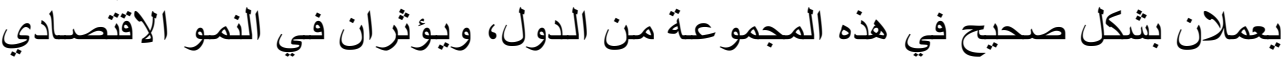

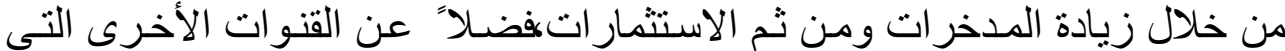

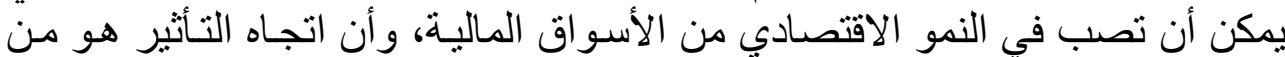

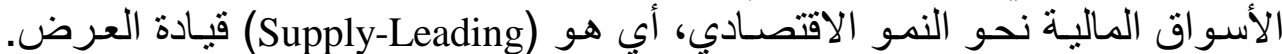

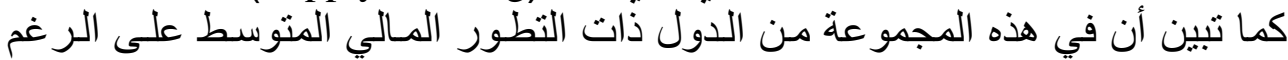
من عدم وجود علاقة ذات شكل قوي بين الأسواق المالية ذاتهاإلا أن كل سوق يعمل بحد ذاته في زيادة النمو الاقتصادي.

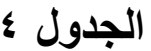

اختبار VAR للاول ذات ذات التطور المالي المتوسط

\begin{tabular}{|c|c|c|c|}
\hline & CB & EQ2 & GG \\
\hline $\mathrm{CB}(-1)$ & -0.051398 & 10.16351 & 0.727705 \\
\hline & $(0.68911)$ & $(23.6819)$ & $(0.33935)$ \\
\hline & $(-0.07459)$ & $(0.42917)$ & $(2.14441)^{*}$ \\
\hline $\mathrm{CB}(-2)$ & -0.329693 & -1.642273 & 0.680387 \\
\hline & $(0.28952)$ & $(9.94954)$ & $(0.14257)$ \\
\hline & $(-1.13878)$ & $(-0.16506)$ & $(4.77225)^{*}$ \\
\hline $\mathrm{CB}(-3)$ & 0.720381 & -3.724644 & -0.641175 \\
\hline & $(0.38981)$ & $(13.3964)$ & $(0.19196)$ \\
\hline & $(1.84802)$ & $(-0.27803)$ & $(-3.34010)^{*}$ \\
\hline $\mathrm{CB}(-4)$ & -0.534287 & 0.633480 & -0.021876 \\
\hline & $(0.38672)$ & $(13.2902)$ & $(0.19044)$ \\
\hline & $(-1.38157)$ & $(0.04767)$ & $(-0.11487)$ \\
\hline $\mathrm{CB}(-5)$ & -0.289989 & 0.706648 & 0.470174 \\
\hline & $(0.43943)$ & $(15.1017)$ & $(0.21640)$ \\
\hline & $(-0.65991)$ & $(0.04679)$ & $(2.17272)^{*}$ \\
\hline $\mathrm{CB}(-6)$ & -0.125840 & 0.873359 & -0.152861 \\
\hline & $(0.28339)$ & $(9.73889)$ & $(0.13955)$ \\
\hline & $(-0.44406)$ & $(0.08968)$ & $(-1.09536)$ \\
\hline \multicolumn{3}{|c}{}
\end{tabular}

\begin{tabular}{|l|c|c|c|}
\hline & CB & EQ2 & GG \\
\hline $\mathrm{CB}(-7)$ & -0.625079 & 3.163596 & 0.927312 \\
\hline & $(0.39070)$ & $(13.4269)$ & $(0.19240)$ \\
\hline & $(-1.59989)$ & $(0.23562)$ & $(4.81971)^{\star *}$ \\
\hline $\mathrm{CB}(-8)$ & 0.395845 & 2.913650 & 0.011352 \\
\hline & $(0.27254)$ & $(9.36627)$ & $(0.13421)$ \\
\hline & $(1.45241)$ & $(0.31108)$ & $(0.08458)$ \\
\hline $\mathrm{CB}(-9)$ & 0.040042 & -5.060382 & -0.264665 \\
\hline & $(0.34338)$ & $(11.8005)$ & $(0.16909)$ \\
\hline & $(0.11661)$ & $(-0.42883)$ & $(-1.56518)$ \\
\hline $\mathrm{CB}(-10)$ & 0.449862 & -1.740106 & -0.043569 \\
\hline & $(0.18639)$ & $(6.40546)$ & $(0.09179)$ \\
\hline & $(2.41357)$ & $(-0.27166)$ & $(-0.47467)$ \\
\hline $\mathrm{CB}(-11)$ & -0.086330 & -3.187946 & -0.768205 \\
\hline & $(0.28600)$ & $(9.82887)$ & $(0.14084)$ \\
\hline & $(-0.30185)$ & $(-0.32435)$ & $(-5.45436)^{* *}$ \\
\hline $\mathrm{CB}(-12)$ & -0.227710 & 3.964384 & 0.661444 \\
\hline & $(0.37448)$ & $(12.8693)$ & $(0.18441)$ \\
\hline & $(-0.60808)$ & $(0.30805)$ & $(3.58680)^{* *}$ \\
\hline
\end{tabular}




\begin{tabular}{|c|c|c|c|}
\hline & $\mathrm{CB}$ & EQ2 & GG \\
\hline \multirow[t]{3}{*}{ EQ2(-1) } & 0.009173 & -0.195809 & -0.015831 \\
\hline & $(0.02310)$ & $(0.79393)$ & $(0.01138)$ \\
\hline & $(0.39706)$ & $(-0.24663)$ & $(-1.39150)$ \\
\hline \multirow{3}{*}{ EQ2(-2) } & -0.058315 & -0.292240 & -0.022692 \\
\hline & $(0.02281)$ & $(0.78392)$ & $(0.01123)$ \\
\hline & $(-2.55649)^{*}$ & $(-0.37279)$ & $(-2.02014)^{*}$ \\
\hline \multirow[t]{3}{*}{ EQ2(-3) } & -0.074623 & 0.610324 & 0.126108 \\
\hline & $(0.07922)$ & (2.72252) & $(0.03901)$ \\
\hline & $(-0.94196)$ & $(0.22418)$ & $(3.23252)^{* *}$ \\
\hline \multirow[t]{3}{*}{ EQ2(-4) } & -0.007411 & 0.166357 & 0.068495 \\
\hline & $(0.03812)$ & $(1.30989)$ & $(0.01877)$ \\
\hline & $(-0.19445)$ & $(0.12700)$ & $(3.64919)^{\star *}$ \\
\hline \multirow[t]{3}{*}{ EQ2(-5) } & -0.231954 & -0.921937 & -0.133100 \\
\hline & $(0.10468)$ & (3.59749) & $(0.05155)$ \\
\hline & $(-2.21581)^{*}$ & $(-0.25627)$ & $(-2.58195)^{*}$ \\
\hline \multirow[t]{3}{*}{ EQ2(-6) } & 0.183207 & 3.356778 & 0.363414 \\
\hline & $(0.26130)$ & (8.97998) & $(0.12868)$ \\
\hline & $(0.70113)$ & $(0.37381)$ & $(2.82420)^{\star}$ \\
\hline \multirow{3}{*}{ EQ2(-7) } & -0.050825 & -2.807667 & -0.122792 \\
\hline & $(0.18318)$ & (6.29534) & $(0.09021)$ \\
\hline & $(-0.27745)$ & $(-0.44599)$ & $(-1.36120)$ \\
\hline \multirow[t]{3}{*}{ EQ2(-8) } & 0.072946 & 0.048172 & -0.030364 \\
\hline & $(0.16037)$ & $(5.51127)$ & $(0.07897)$ \\
\hline & $(0.45486)$ & $(0.00874)$ & $(-0.38449)$ \\
\hline \multirow[t]{3}{*}{ EQ2(-9) } & -0.250961 & 0.946942 & 0.076311 \\
\hline & $(0.13791)$ & $(4.73937)$ & $(0.06791)$ \\
\hline & $(-1.81977)^{\star}$ & $(0.19980)$ & $(1.12366)$ \\
\hline \multirow[t]{3}{*}{ EQ2(-10) } & -0.062604 & 1.187644 & 0.349186 \\
\hline & $(0.14215)$ & $(4.88506)$ & $(0.07000)$ \\
\hline & $(-0.44042)$ & $(0.24312)$ & $(4.98835)^{\star \star}$ \\
\hline \multirow[t]{3}{*}{ EQ2(-11) } & -0.056816 & 0.163431 & -0.296997 \\
\hline & $(0.13690)$ & (4.70473) & $(0.06742)$ \\
\hline & $(-0.41501)$ & $(0.03474)$ & $(-4.40541)^{\star *}$ \\
\hline \multirow[t]{3}{*}{ EQ2(-12) } & -0.226283 & 3.921316 & 0.506131 \\
\hline & $(0.28722)$ & (9.87048) & $(0.14144)$ \\
\hline & $(-0.78785)$ & $(0.39728)$ & $(3.57845)^{\star *}$ \\
\hline \multirow[t]{3}{*}{$\mathrm{GG}(-1)$} & -0.924026 & 4.722970 & 1.653068 \\
\hline & $(0.59110)$ & $(20.3138)$ & $(0.29109)$ \\
\hline & $(-1.56323)$ & $(0.23250)$ & $(5.67897)$ \\
\hline \multirow[t]{3}{*}{$G G(-2)$} & -0.169102 & -1.366213 & 0.080182 \\
\hline & $(0.31629)$ & $(10.8696)$ & $(0.15575)$ \\
\hline & $(-0.53465)$ & $(-0.12569)$ & $(0.51480)$ \\
\hline \multirow[t]{3}{*}{$\mathrm{GG}(-3)$} & -0.779001 & 6.913461 & 0.209603 \\
\hline & $(0.41302)$ & (14.1938) & $(0.20339)$ \\
\hline & $(-1.88613)$ & $(0.48708)$ & $(1.03055)$ \\
\hline \multirow[t]{3}{*}{$G G(-4)$} & -1.700981 & 6.847355 & 1.225661 \\
\hline & $(0.51320)$ & (17.6368) & $(0.25273)$ \\
\hline & $(-3.31444)^{\star *}$ & (0.38824) & $(4.84975)^{\star *}$ \\
\hline
\end{tabular}

\begin{tabular}{|l|c|c|c|}
\hline & CB & EQ2 & GG \\
\hline sGG(-5) & -1.763446 & 16.09433 & 2.013970 \\
\hline & $(1.21966)$ & $(41.9150)$ & $(0.60062)$ \\
\hline & $(-1.44585)$ & $(0.38398)$ & $(3.35316)^{* \star}$ \\
\hline GG(-6) & -2.564540 & 14.15973 & 1.618964 \\
\hline & $(1.14813)$ & $(39.4568)$ & $(0.56540)$ \\
\hline & $(-2.23367)^{*}$ & $(0.35887)$ & $(2.86342)^{\star}$ \\
\hline GG(-7) & -1.665049 & 20.22542 & 2.821015 \\
\hline & $(1.72628)$ & $(59.3256)$ & $(0.85011)$ \\
\hline & $(-0.96453)$ & $(0.34092)$ & $(3.31843)^{\star *}$ \\
\hline GG(-8) & -0.396241 & 1.926303 & 1.208978 \\
\hline & $(0.59009)$ & $(20.2790)$ & $(0.29059)$ \\
\hline & $(-0.67150)$ & $(0.09499)$ & $(4.16046)^{\star *}$ \\
\hline GG(-9) & 0.494672 & -4.284305 & 0.055695 \\
\hline & $(0.25531)$ & $(8.77416)$ & $(0.12573)$ \\
\hline & $(1.93751)^{*}$ & $(-0.48829)$ & $(0.44297)$ \\
\hline GG(-10) & 0.160794 & -4.808431 & -0.674541 \\
\hline & $(0.35709)$ & $(12.2720)$ & $(0.17585)$ \\
\hline & $(0.45029)$ & $(-0.39182)$ & $(-3.83588)^{* *}$ \\
\hline GG(-11) & -0.028871 & -5.700334 & -0.276534 \\
\hline & $(0.39744)$ & $(13.6586)$ & $(0.19572)$ \\
\hline & $(-0.07264)$ & $(-0.41735)$ & $(-1.41291)$ \\
\hline GG(-12) & -0.042179 & -0.225654 & 0.504941 \\
\hline & $(0.46380)$ & $(15.9391)$ & $(0.22840)$ \\
\hline & $(-0.09094)$ & $(-0.01416)$ & $(2.21078)^{\star}$ \\
\hline C & 172.7190 & -850.8813 & -169.3346 \\
\hline & $(114.294)$ & $(3927.85)$ & $(56.2840)$ \\
\hline & $(1.51118)$ & $(-0.21663)$ & $(-3.00857)^{* *}$ \\
\hline R- ${ }^{2}$ & 0.999269 & 0.854545 & 0.997674 \\
\hline Adj. R- ${ }^{2}$ & 0.986102 & -1.763651 & 0.955811 \\
\hline S.E. & 2.347036 & 80.65858 & 1.155795 \\
\hline F- & 75.89464 & 0.326387 & 23.83183 \\
\hline Log & -30.68849 & -168.6343 & -3.062267 \\
likelihood & & & \\
\hline AIC & 3.471205 & 10.54535 & 2.054475 \\
\hline SC & 5.049456 & 12.12360 & 3.632726 \\
\hline & & \\
\hline & &
\end{tabular}

$*, * *$, Indicate significance at the $5 \%, 1 \%$ level

High Financial Development States 


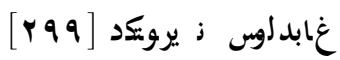

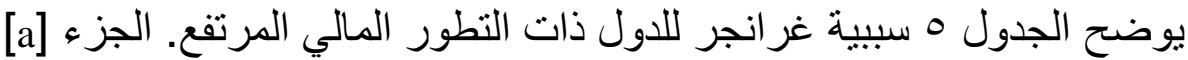

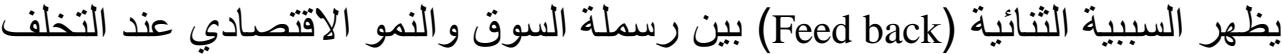

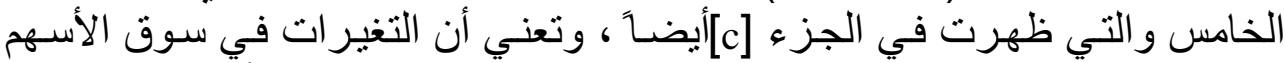

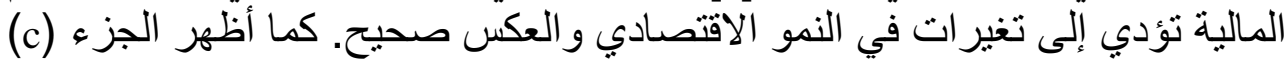

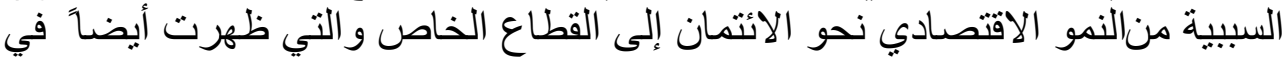

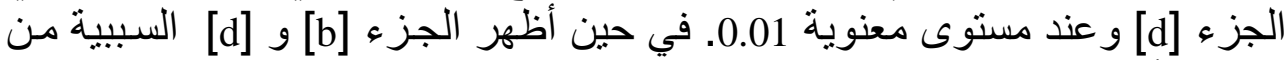

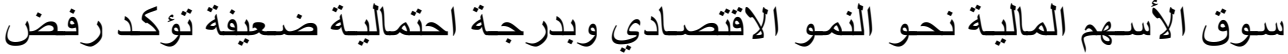

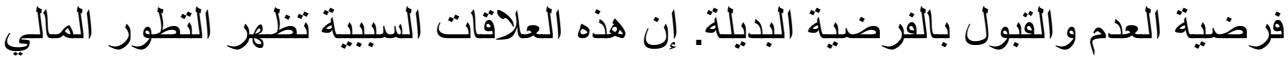

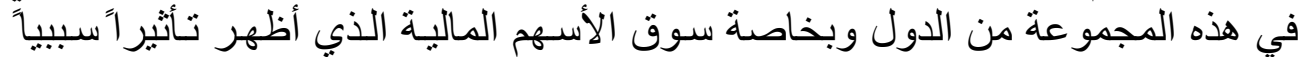
ثنائياً (Feed back) مع النمو الاقتصادي.

[a]

\begin{tabular}{|c|c|c|}
\hline \multicolumn{3}{|l|}{ gs: 5} \\
\hline & F-Statistic & Probability \\
\hline $\mathrm{EQ} 1 \rightarrow \mathrm{CB}$ & 0.08980 & 0.99347 \\
\hline $\mathrm{CB} \rightarrow \mathrm{EQ} 1$ & 0.75976 & 0.58355 \\
\hline $\mathrm{GG} \rightarrow \mathrm{CB}$ & 1.57395 & 0.18418 \\
\hline $\mathrm{CB} \rightarrow \mathrm{GG}$ & 1.17996 & 0.33197 \\
\hline $\mathrm{GG} \rightarrow \mathrm{EQ1}$ & $3.70572^{*}$ & 0.00607 \\
\hline $\mathrm{EQ} 1 \rightarrow \mathrm{GG}$ & $2.72715^{*}$ & 0.02914 \\
\hline
\end{tabular}

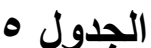

\section{اختبار سبيية غرانجر للاول ذات التطور المالي المرتفع}

[b]

\begin{tabular}{|c|c|c|}
\hline \multicolumn{3}{|l|}{ Lags: 5} \\
\hline & F-Statistic & Probability \\
\hline $\mathrm{EQ} 2 \rightarrow \mathrm{CB}$ & 0.16669 & 0.97275 \\
\hline $\mathrm{CB} \rightarrow \mathrm{EQ} 2$ & 0.93653 & 0.47265 \\
\hline $\mathrm{GG} \rightarrow \mathrm{CB}$ & 1.57395 & 0.18418 \\
\hline $\mathrm{CB} \rightarrow \mathrm{GG}$ & 1.17996 & 0.33197 \\
\hline $\mathrm{GG} \rightarrow \mathrm{EQ} 2$ & $2.74013^{*}$ & 0.03885 \\
\hline $\mathrm{EQ} 2 \rightarrow \mathrm{GG}$ & 0.38546 & 0.85449 \\
\hline
\end{tabular}

[c]

\begin{tabular}{|l|c|c|}
\hline \multicolumn{3}{|l|}{ Lags: 5} \\
\hline & F-Statistic & Probability \\
\hline $\mathrm{EQ} 1 \rightarrow \mathrm{CP}$ & 0.19190 & 0.96411 \\
\hline $\mathrm{CP} \rightarrow \mathrm{EQ} 1$ & 0.39220 & 0.85157 \\
\hline $\mathrm{GG} \rightarrow \mathrm{CP}$ & $3.99742^{\star *}$ & 0.00390 \\
\hline $\mathrm{CP} \rightarrow \mathrm{GG}$ & 0.56454 & 0.72665 \\
\hline $\mathrm{GG} \rightarrow \mathrm{EQ} 1$ & $3.70572^{\star *}$ & 0.00607 \\
\hline $\mathrm{EQ} 1 \rightarrow \mathrm{GG}$ & $2.72715^{\star}$ & 0.02914 \\
\hline
\end{tabular}

[d]

\begin{tabular}{|l|c|c|}
\hline Lags: 5 & \multicolumn{1}{l|}{} \\
\hline & F-Statistic & Probability \\
\hline $\mathrm{EQ} 2 \rightarrow \mathrm{CP}$ & 0.07793 & 0.99513 \\
\hline $\mathrm{CP} \rightarrow \mathrm{EQ} 2$ & 0.58206 & 0.71340 \\
\hline $\mathrm{GG} \rightarrow \mathrm{CP}$ & $3.99742^{\star *}$ & 0.00390 \\
\hline $\mathrm{CP} \rightarrow \mathrm{GG}$ & 0.56454 & 0.72665 \\
\hline $\mathrm{GG} \rightarrow \mathrm{EQ} 2$ & $2.74013^{*}$ & 0.03885 \\
\hline $\mathrm{EQ} 2 \rightarrow \mathrm{GG}$ & 0.38546 & 0.85449 \\
\hline
\end{tabular}

أما تحليل الانحدار الذاتي للمتجهة فيظهر في الجدول 7 لمجموعة الدول ذات

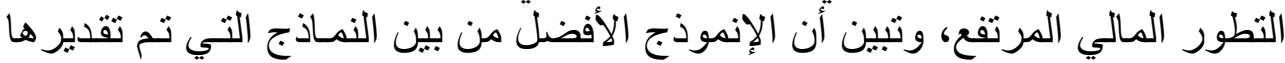

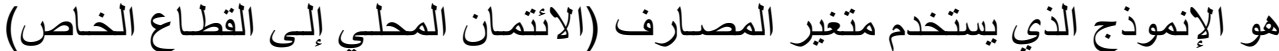

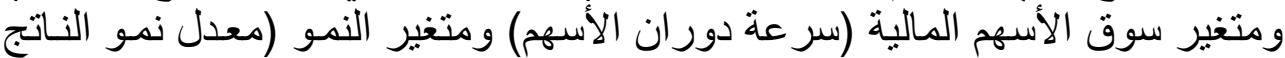
المحلي الإجمالي لكل فرد)، و أفضل تخلف للإنموذج وحسب الإنب أقل قيمة لمعيار شوارز (SC)

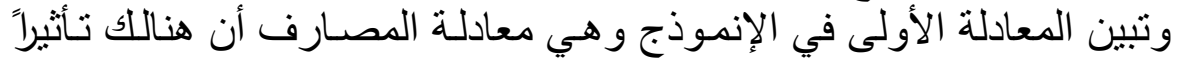

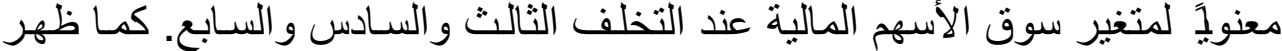

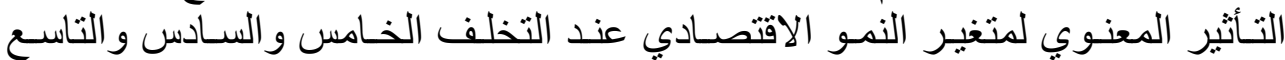
وبجمع معلمات متغير النمو مع بقاء العو امل الأخرى ثابتـة نجد أن زيـادة نمو النـاتج 


$$
\begin{aligned}
& \text { يد بحتة قومنلكا تقيلمه ا قا وسلأ ا... } \\
& \text { [r...] }
\end{aligned}
$$

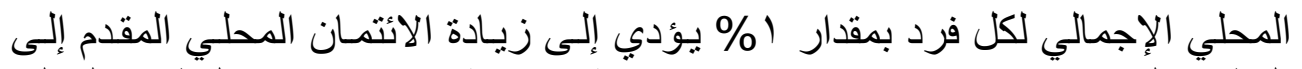

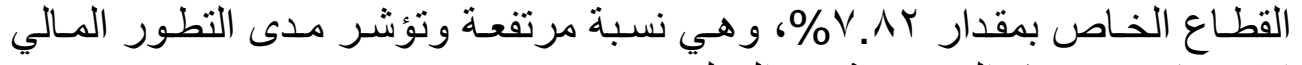
الاقتصادي في هذه المجمو عة من الدول.

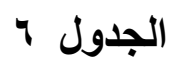

اختبار VAR للاول ذات ذات التطور المالي المرتفع 
غابدلوس ز يروتيكد [1 +r]

\begin{tabular}{|c|c|c|c|}
\hline & $\mathrm{CP}$ & EQ2 & GG \\
\hline \multirow[t]{3}{*}{$\mathrm{CP}(-1)$} & 0.923971 & 3.435251 & 0.050530 \\
\hline & $(0.21358)$ & $(1.51511)$ & $(0.12391)$ \\
\hline & $(4.32604)^{*}$ & $(2.26732)^{*}$ & $(0.40781)$ \\
\hline \multirow[t]{3}{*}{$\mathrm{CP}(-2)$} & -0.055174 & 1.040616 & 0.101748 \\
\hline & $(0.27579)$ & $(1.95636)$ & $(0.15999)$ \\
\hline & $(-0.20006)$ & $(0.53191)$ & $(0.63595)$ \\
\hline \multirow[t]{3}{*}{$\mathrm{CP}(-3)$} & -0.573090 & -4.222058 & -0.138150 \\
\hline & $(0.21025)$ & $(1.49149)$ & $(0.12197)$ \\
\hline & $(-2.72571)^{*}$ & $(-2.83077)^{\star}$ & $(-1.13262)$ \\
\hline \multirow[t]{3}{*}{$\mathrm{CP}(-4)$} & 0.179715 & -1.707775 & -0.180292 \\
\hline & $(0.21693)$ & $(1.53884)$ & $(0.12585)$ \\
\hline & $(0.82846)$ & $(-1.10978)$ & $(-1.43263)$ \\
\hline \multirow[t]{3}{*}{$\mathrm{CP}(-5)$} & 0.598024 & 1.659622 & 0.267420 \\
\hline & $(0.19723)$ & $(1.39908)$ & $(0.11442)$ \\
\hline & $(3.03217)^{\star *}$ & $(1.18622)$ & $(2.33723)^{*}$ \\
\hline \multirow[t]{3}{*}{$\mathrm{CP}(-6)$} & -0.309477 & -1.983043 & -0.029028 \\
\hline & $\begin{array}{l}(0.17191) \\
\end{array}$ & $(1.21948)$ & $(0.09973)$ \\
\hline & $(-1.80024)^{\star}$ & $(-1.62614)$ & $(-0.29107)$ \\
\hline \multirow[t]{3}{*}{$\mathrm{CP}(-7)$} & 0.118267 & -0.241580 & -0.095641 \\
\hline & $(0.17696)$ & $(1.25529)$ & $(0.10266)$ \\
\hline & $(0.66834)$ & $(-0.19245)$ & $(-0.93164)$ \\
\hline \multirow[t]{3}{*}{$\mathrm{CP}(-8)$} & -0.031064 & -0.301448 & 0.029982 \\
\hline & $(0.11958)$ & $(0.84828)$ & $(0.06937)$ \\
\hline & $(-0.25978)$ & $(-0.35536)$ & $(0.43219)$ \\
\hline \multirow[t]{3}{*}{$\mathrm{CP}(-9)$} & -0.036654 & 1.002993 & 0.210113 \\
\hline & $(0.12482)$ & $(0.88546)$ & $(0.07241)$ \\
\hline & $(-0.29365)$ & $(1.13274)$ & $(2.90157)^{\star}$ \\
\hline \multirow[t]{3}{*}{ EQ2(-1) } & 0.024723 & -0.695165 & -0.022234 \\
\hline & $(0.05765)$ & $(0.40897)$ & $(0.03345)$ \\
\hline & $(0.42883)$ & $(-1.69980)$ & $(-0.66478)$ \\
\hline \multirow[t]{3}{*}{ EQ2(-2) } & 0.094598 & 0.577507 & 0.063912 \\
\hline & $(0.05668)$ & $(0.40210)$ & $(0.03288)$ \\
\hline & $(1.66886)$ & $(1.43621)$ & $(1.94354)^{\star}$ \\
\hline \multirow[t]{3}{*}{ EQ2(-3) } & 0.108111 & 0.262007 & 0.012093 \\
\hline & $(0.05476)$ & $(0.38845)$ & $(0.03177)$ \\
\hline & $(1.97431)^{*}$ & $(0.67450)$ & $(0.38067)$ \\
\hline \multirow[t]{3}{*}{ EQ2(-4) } & -0.145304 & 1.286764 & 0.124965 \\
\hline & $(0.10658)$ & $(0.75605)$ & $(0.06183)$ \\
\hline & $(-1.36334)$ & $(1.70196)$ & $(2.02110)^{\star}$ \\
\hline \multirow[t]{3}{*}{ EQ2(-5) } & 0.034449 & 0.135536 & -0.100112 \\
\hline & $(0.06154)$ & $(0.43658)$ & $(0.03570)$ \\
\hline & $(0.55975)$ & $(0.31045)$ & $\begin{array}{c}(- \\
2.80396)^{*} \\
\end{array}$ \\
\hline \multirow[t]{3}{*}{ EQ2(-6) } & -0.134490 & -0.509930 & -0.034150 \\
\hline & $(0.03783)$ & $(0.26833)$ & $(0.02194)$ \\
\hline & $(-3.55552)^{\star *}$ & $(-1.90040)^{*}$ & $(-1.55622)$ \\
\hline \multirow[t]{3}{*}{ EQ2(-7) } & -0.222605 & -0.469079 & -0.031846 \\
\hline & $(0.04484)$ & $(0.31808)$ & $(0.02601)$ \\
\hline & $(-4.96454)^{* *}$ & $(-1.47474)$ & $(-1.22424)$ \\
\hline EQ2(-8) & 0.068552 & 0.847921 & 0.028299 \\
\hline
\end{tabular}

\begin{tabular}{|c|c|c|c|}
\hline & $\mathrm{CP}$ & EQ2 & GG \\
\hline & $(0.05623)$ & $(0.39888)$ & $(0.03262)$ \\
\hline & $(1.21915)$ & $(2.12577)^{*}$ & $(0.86754)$ \\
\hline \multirow[t]{3}{*}{ EQ2(-9) } & -0.010983 & 1.207225 & 0.084384 \\
\hline & $(0.08500)$ & $(0.60295)$ & $(0.04931)$ \\
\hline & $(-0.12921)$ & $(2.00219)^{*}$ & $(1.71130)$ \\
\hline \multirow{3}{*}{$\mathrm{GG}(-1)$} & 0.472273 & -12.13338 & -1.119760 \\
\hline & $(0.59879)$ & $(4.24766)$ & $(0.34738)$ \\
\hline & $(0.78872)$ & $(-2.85649)^{*}$ & $(-3.2234)^{\star \star}$ \\
\hline \multirow[t]{3}{*}{$\mathrm{GG}(-2)$} & -0.712875 & -14.22811 & -0.902443 \\
\hline & $(0.69227)$ & $(4.91083)$ & $(0.40161)$ \\
\hline & $(-1.02976)$ & $(-2.89729)^{*}$ & $\begin{array}{c}(- \\
2.24706)^{*}\end{array}$ \\
\hline \multirow[t]{3}{*}{$\mathrm{GG}(-3)$} & -0.507083 & -12.46040 & -0.113165 \\
\hline & $(0.55563)$ & $(3.94150)$ & $(0.32234)$ \\
\hline & $(-0.91263)$ & $(-3.16133)^{\star *}$ & $(-0.35107)$ \\
\hline \multirow[t]{3}{*}{$G G(-4)$} & 0.710906 & -5.448279 & 0.096408 \\
\hline & $(0.67805)$ & $(4.80996)$ & $(0.39336)$ \\
\hline & $(1.04845)$ & $(-1.13271)$ & $(0.24509)$ \\
\hline \multirow[t]{3}{*}{ GG(-5) } & 5.118457 & 28.21919 & 2.609626 \\
\hline & $(2.87476)$ & (20.3929) & $(1.66774)$ \\
\hline & $(1.78048)$ & $(1.38378)$ & $(1.56477)$ \\
\hline \multirow[t]{3}{*}{$\mathrm{GG}(-6)$} & -5.051527 & -48.46472 & -1.992761 \\
\hline & $(2.20811)$ & (15.6638) & $(1.28100)$ \\
\hline & $(-2.28771)^{*}$ & $(-3.09405)^{*}$ & $(-1.55563)$ \\
\hline \multirow[t]{3}{*}{$\mathrm{GG}(-7)$} & 2.352997 & -23.03746 & -0.905277 \\
\hline & $(1.73914)$ & $(12.3370)$ & (1.00893) \\
\hline & $(1.35297)$ & $(-1.86734)^{*}$ & $(-0.89727)$ \\
\hline \multirow[t]{3}{*}{$\mathrm{GG}(-8)$} & 1.129674 & -29.91014 & -0.919742 \\
\hline & $(2.08683)$ & $(14.8035)$ & $(1.21064)$ \\
\hline & $(0.54133)$ & $(-2.02048)^{*}$ & $(-0.75972)$ \\
\hline \multirow[t]{3}{*}{ GG(-9) } & 4.320825 & 16.18090 & 2.718499 \\
\hline & $(1.44947)$ & $(10.2822)$ & $(0.84088)$ \\
\hline & $(2.98097)^{*}$ & $(1.57368)$ & $(3.2329)^{\star \star}$ \\
\hline \multirow[t]{3}{*}{$C$} & -9.780446 & 559.6261 & -28.72378 \\
\hline & (27.6538) & $(196.170)$ & $(16.0429)$ \\
\hline & $(-0.35367)$ & $(2.85277)^{*}$ & $(-1.79044)$ \\
\hline$R^{2}-$ & 0.996774 & 0.980935 & 0.931588 \\
\hline Adj. $\mathrm{R}^{2}{ }^{2}$ & 0.967735 & 0.809354 & 0.315877 \\
\hline S.E. & 6.030441 & 42.77853 & 3.498454 \\
\hline F- & 34.32610 & 5.717020 & 1.513029 \\
\hline $\begin{array}{l}\text { Log } \\
\text { likelihood }\end{array}$ & -63.49021 & -124.2259 & -46.61073 \\
\hline AIC & 5.902594 & 9.821026 & 4.813596 \\
\hline SC & 7.197808 & 11.11624 & 6.108810 \\
\hline \multicolumn{2}{|c|}{$\begin{array}{l}\text { Determinant Residual } \\
\text { Covariance }\end{array}$} & 386.1684 & \\
\hline \multicolumn{2}{|c|}{ Log Likelihood } & -224.2835 & \\
\hline \multicolumn{2}{|c|}{$\begin{array}{l}\text { Akaike Information } \\
\text { Criteria }\end{array}$} & 19.88926 & \\
\hline \multicolumn{2}{|c|}{ Schwarz Criteria } & 23.77490 & \\
\hline
\end{tabular}

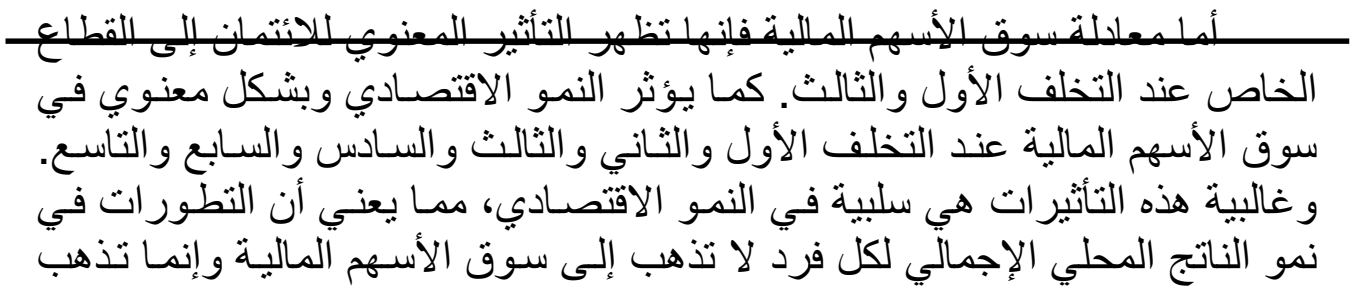


إلى النمو في الائتمان والقطاع المصرفي، أو أن النمو يؤدي إلى تحجيم التوجيـه إلى الـى

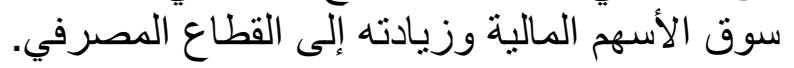

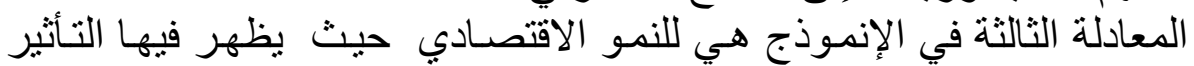

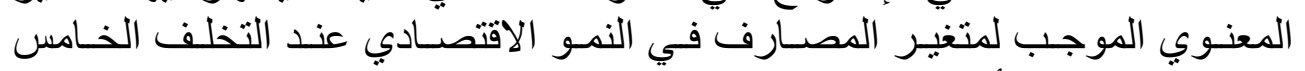

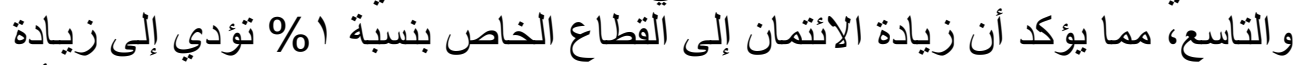

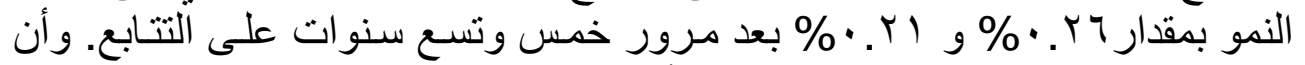

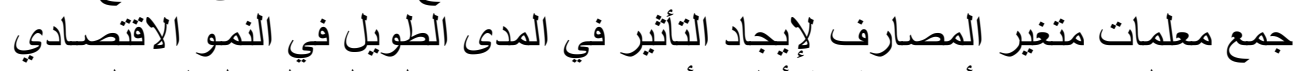

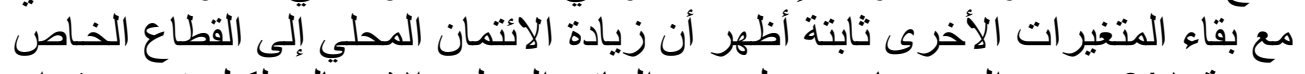

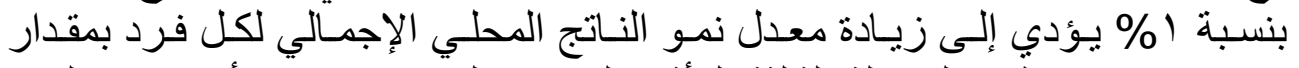

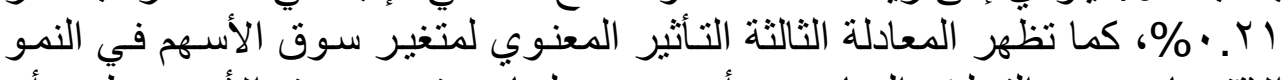

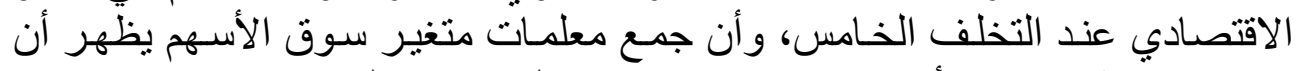
زيادة سرعة دوران الأسهم بمقدار ل (\% يؤدي إلى زيادة النمو الاقتصـادي بمقدار

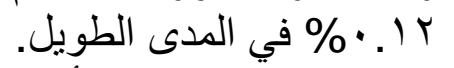

مما سبق نستنتج أنه على الرغم من أن سبيلية غر انجر أظهرت السبيلية الثنائية

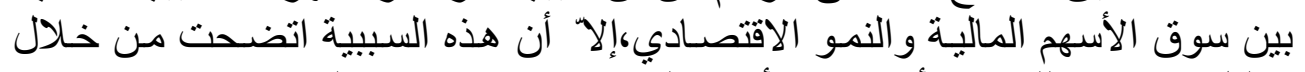

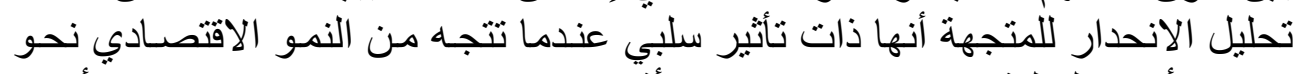

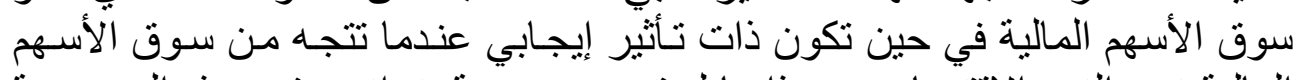

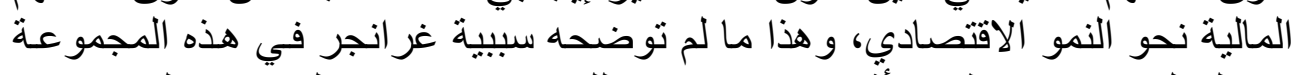

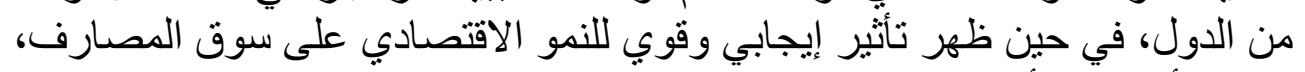

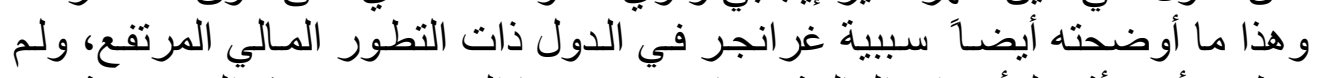

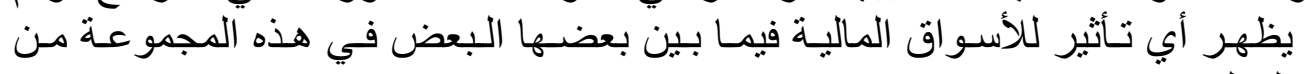

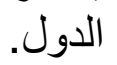

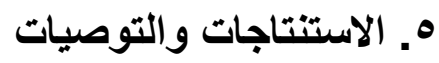

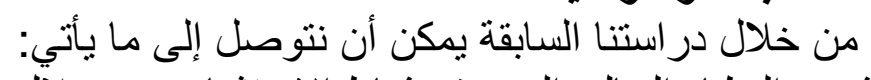

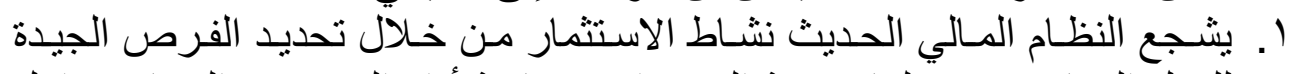

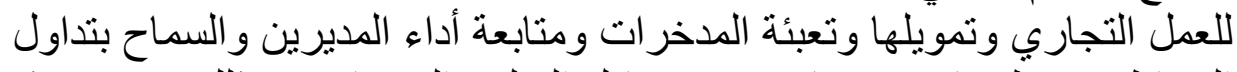

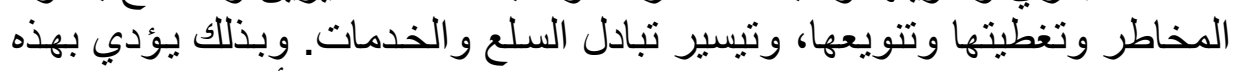

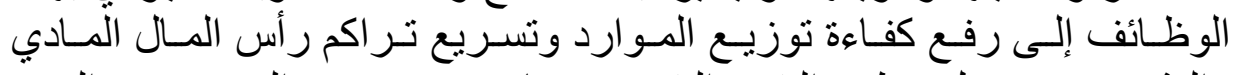

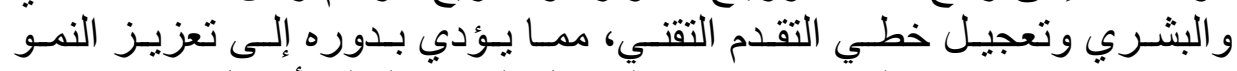

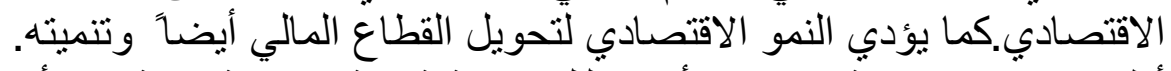

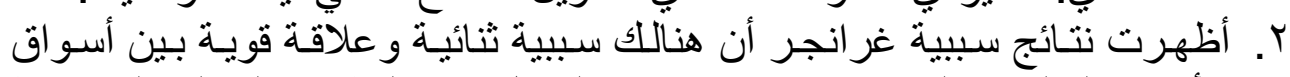

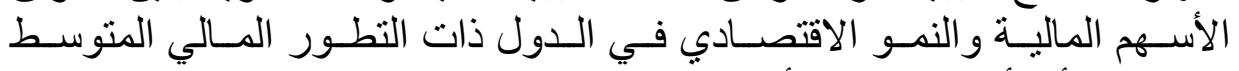

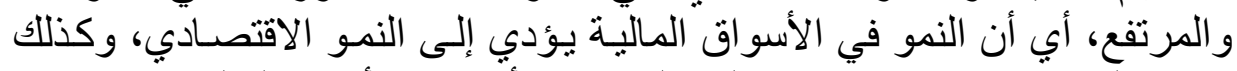

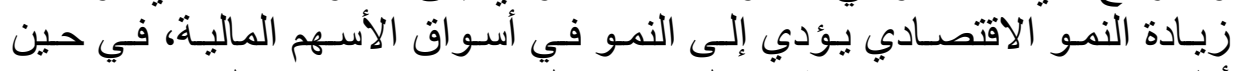

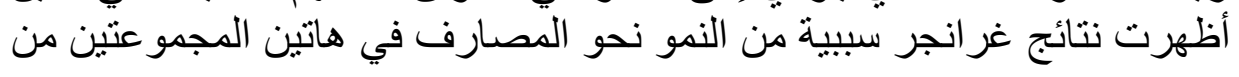


غابدلوس ; يروتيكد [r.r]

الدول، ولم تظهر أي علاقة سبيية من المصارف إلى النمو، كمـا لم تظهر أي المالي

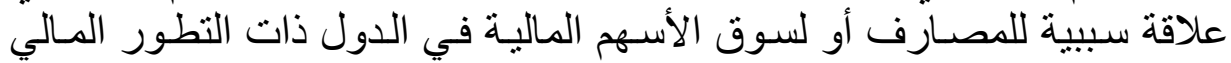

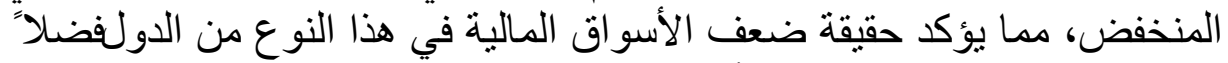

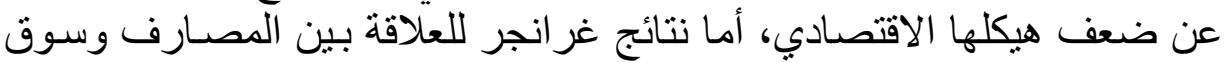

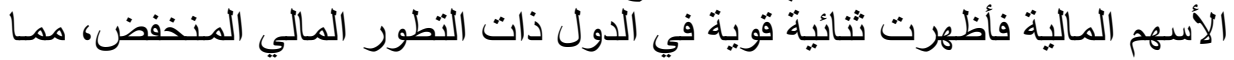

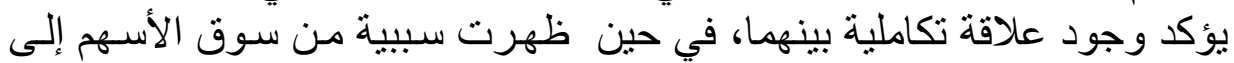

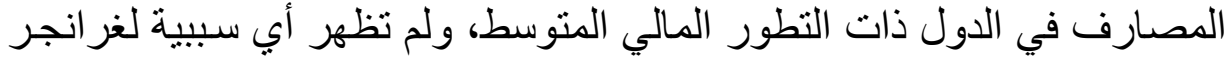

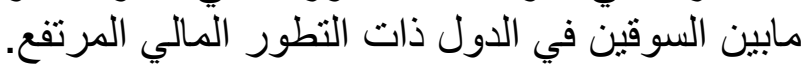

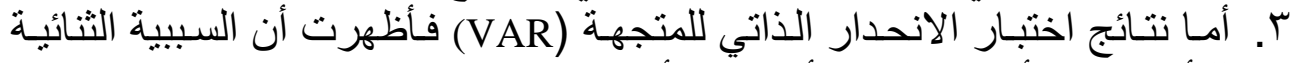

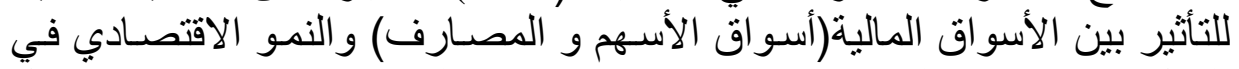

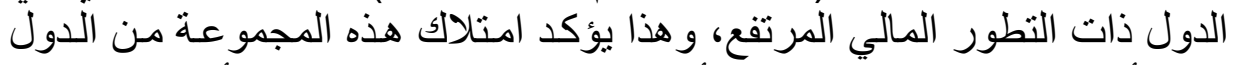

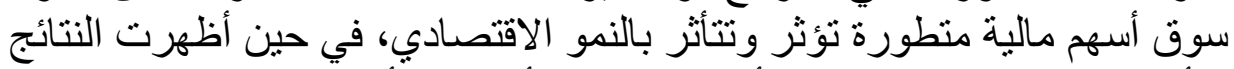

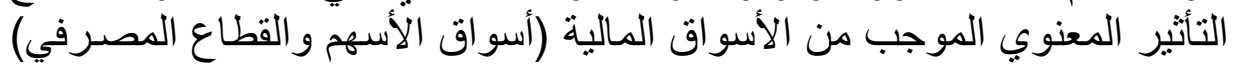

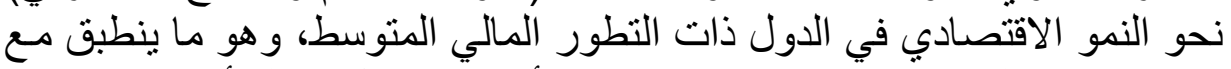

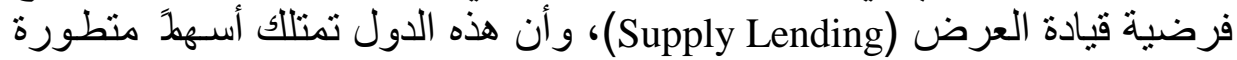

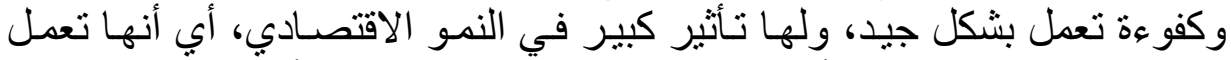

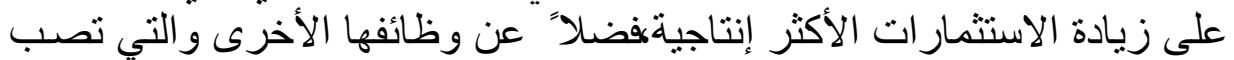

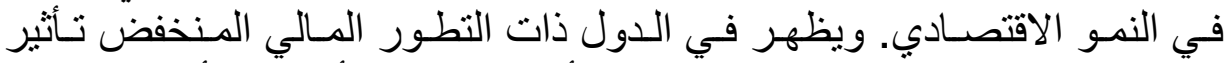

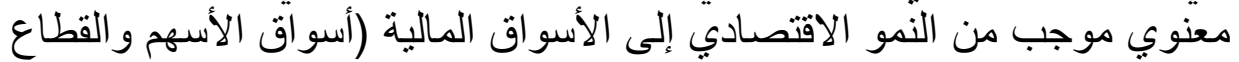

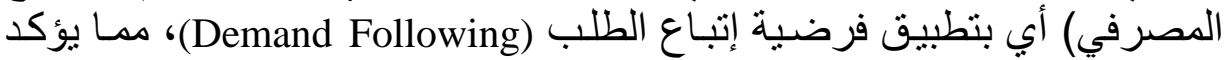
أن هذه الدول هي في بداية تطور ها المبالي المالي وأنها تحتاج إلى النمو الاقتصادي لكي

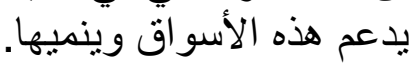

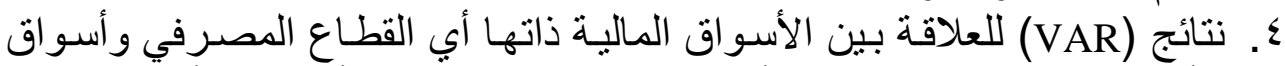

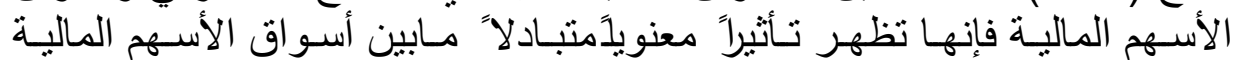

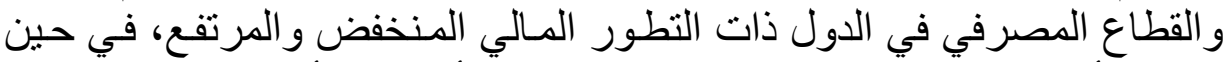

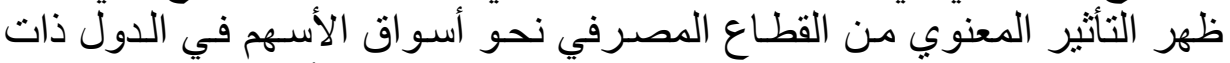

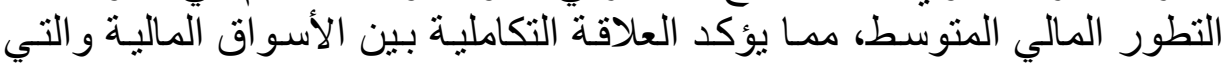

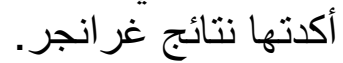

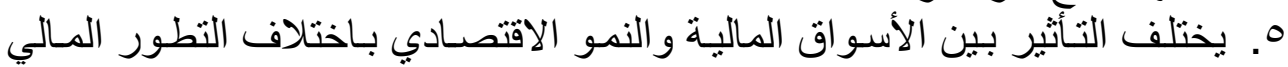

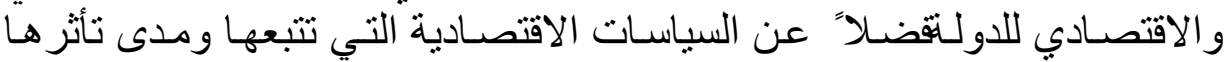

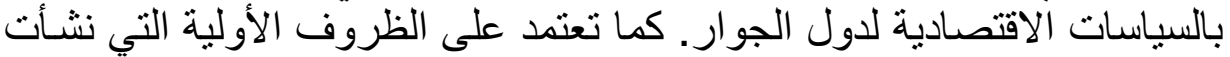

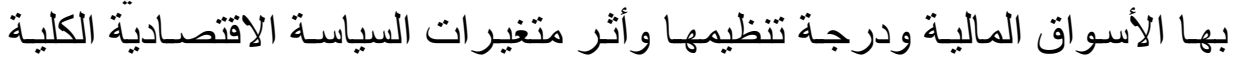

$$
\text { أما أهم التوصيات التي توصل إليها البحث: }
$$

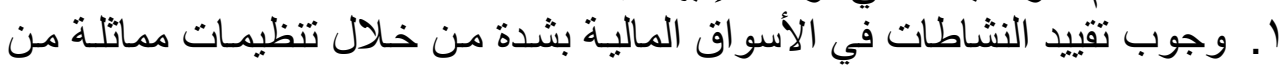

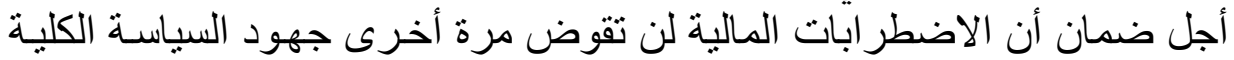

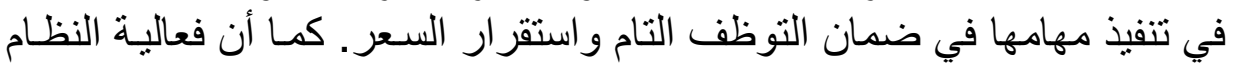




$$
\begin{aligned}
& \text { يدلحسة قاومنكا تقيلمه ا قا وسلأ ا... } \\
& \text { [r. ६] }
\end{aligned}
$$
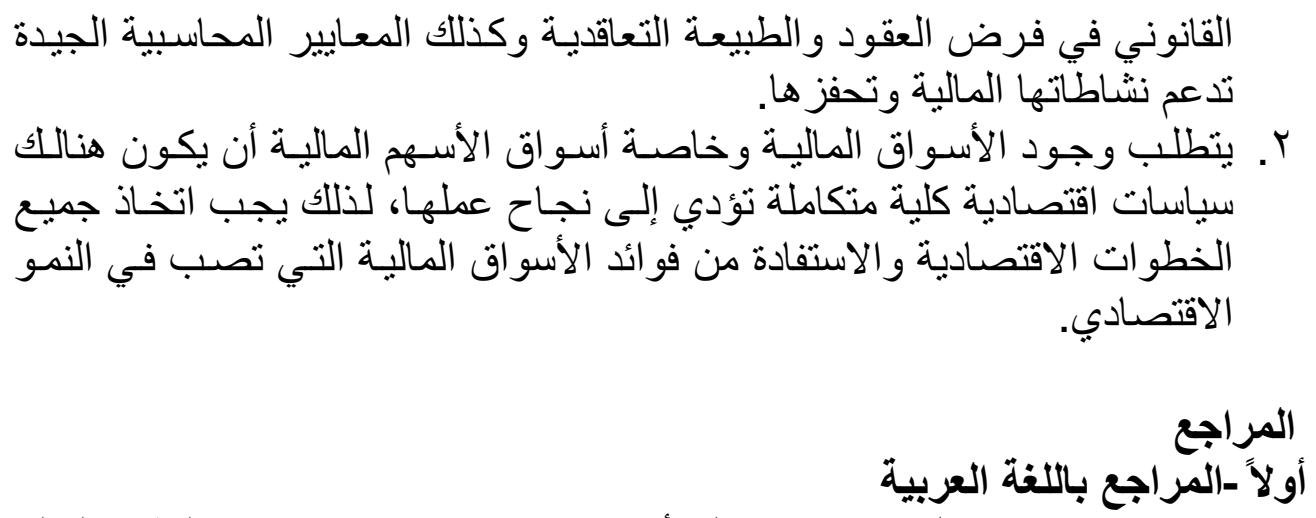

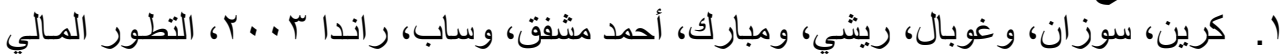

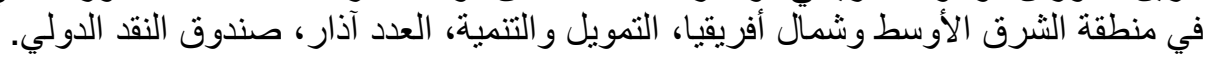

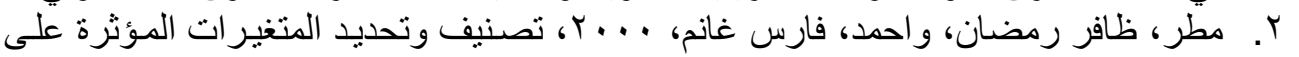

الحمل عند مستويات الخطورة، مجلة النربية والعلم، العددء ؛ ، جامعة الموصل.

\section{ثنانياً -المراجع باللغة الاجنبية}

1. Beck, T. and Levine, R., 2002, "Stock Markets, Bank and Growth: Panel Evidence," NBER, Working paper, 9082, www.nber.org/ papers/w9082.

2. Bennaceur, S., and Ghazouani, S., 2003, "Stock Market, Banks, and Growth in some MENA Region Countries," www.erf.org/.../ Financial_MarketsBackground/Bennaceur Ghazouani.pdf.

3. Boulila, G, and Trabelsi, M., 2002, "Financial Development and Long-Run Growth: Granger Causality in a bivariate VAR structure Evidence from Tunisia: 1962-1997.".

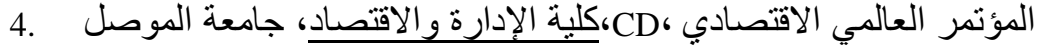

5. Brandl, M., "The Role of Financial Institutions in Long-Run Economic Growth," University of Texas At Austin, www.mccombs, utexas.edu./.../Michael. Brandl/Fin lnst and Long run growth.doc.

6. Eapen, K. 2002" Do Capital Markets Assist Economic Growth?", www.utiicm.com/cmc/pdfs/2002/kveapen^03.pdf.

7. Granger, C., 1969, "Investigating Causal Relations by Econometric Models and CrossSpectral Methods", Econometrica, Vol;37, No:3.

8. King. R. Levine, R., 1993," Finance and Growth: Schumpeter Might be Right", The Quarterly Journal of Economics, 108.

9. Levine, R., 1996," Financial Development and Economic Growth: View and Agenda", policy Research Working paper, 1678, www.worldBank.org/html/dec/publications/workpapers/wps1678-html.

10. Liu, L., and Garcia, R; (1999)," Macroeconomic Determinants of stock Market Development", Journal of Applied Economic, vol: 11, No:1.

11. Lucas, R.(1988)," On the Mechanics of Economic Development", Journal of Monetary Economic, 22, Holland.

12. Greene, w., (2000), "Econometric Analysis", Fourth Edition, New Jersey.

13. Gujarati, D, (1995)," Basic Econometrics", Third Edition, McGraw-Hill, Singapore.

14. World Bank (2004), World Development Indicator (WDI), CD- ROM. 